\title{
HST photometry: Can UV colors probe the galaxy ages?
}

\author{
C. Chiosi ${ }^{1}$, A. Vallenari ${ }^{2}$, and A. Bressan ${ }^{2}$ \\ 1 Department of Astronomy, University of Padua, Vicolo Osservatorio 5, 35122 Padua, Italy \\ 2 Astronomical Observatory, Vicolo Osservatorio 5, 35122, Padua, Italy
}

Received February 12; accepted June 7, 1996

\begin{abstract}
We present grids of magnitudes and colours for the WFPC2 of the refurbished HST using passbands from the far UV to the near infrared, i.e. the filters F170W, F218W, F255W, F300W, F336W, F439W, F450W, F555W, F606W, F702W, F814W, and F850LP. In addition to this, we consider also the filters F150W+F130LP, F175W, F220W and F342W of the old HST in the FOC mode in which a great deal of observations have been taken.
\end{abstract}

The theoretical magnitudes and colours are calculated convolving spectral energy distributions with different metallicity $(Z)$, effective temperature $\left(T_{\text {eff }}\right)$, and gravity with the pass-band transmission, response of the camera, and telescope assembly. In order to enable an easy usage of the present results we provide tabulations of Bolometric Corrections $B C_{\Delta \lambda}^{\mathrm{HST}}$ as function of gravity, $T_{\mathrm{eff}}$, and chemical composition for each pass-band under consideration. As already pointed out by Yi et al. (1995), the UV filters suffer from the visible/red-leak problem, which destroys the monotonic relation between colours and $T_{\text {eff }}$ and causes serious difficulties to analyze the data. With the aid of the above technique we calculate isochrones in the observational colour - magnitude diagram (CMD) and integrated magnitudes and colours of single stellar populations (SSP). The ages span the range from $18 \mathrm{Gyr}$ to 0.01 Gyr. For the sake of brevity, results are described here in some detail limited to the solar chemical composition $[Y=0.28 Z=0.020]$. In addition to this, we present synthetic CMDs for a composite sample of stars containing both young and old objects as they would appear in various pass-bands.

Finally, we address the question whether the variation of the UV colours of elliptical galaxies as a function of the red-shift shows signatures from which one can infer the age and the type of source emitting the UV flux. The goal is achieved looking at the red-shift dependence of colours obtained from the pass-bands F170W, F210W, and F555W at varying the Hubble constant $H_{0}$ and deceleration parameter $q_{0}$ defining the model of the Universe. It is found that those colours run differently according to underlying

Send offprint requests to: C. Chiosi source, i.e either $\mathrm{P}-\mathrm{AGB}$ or $\mathrm{P}-\mathrm{AGB}$ plus $\mathrm{H}-\mathrm{HB}$ and AGBmanqué stars (see below for details) and the cosmological parameters. If the UV flux is mainly generated by $\mathrm{P}-\mathrm{AGB}$ plus H-HB and AGB-manqué stars a marked discontinuity in suitable UV colours is expected at the red-shift corresponding to the age of about 5.6 Gyr.

Key words: techniques: photometry — stars: HR diagram - galaxies: ellipticals — galaxies: evolution ultraviolet: galaxies

\section{Introduction}

The advent of the HST astronomy, which makes available observational data acquired through filters different from those employed in ground-based photometry, requires the correct understanding of how the observations of familiar objects like young and old stars in fields and clusters, stellar populations of different ages and metallicity, etc. would look like in the still poorly known photometric systems of HST. This is particularly true for the UV pass-bands for which the serious problem of the visible/red-leak is known to exist (Nota et al. 1994).

There have been several studies aimed at providing useful calibrations of the HST photometric system. In brief, Harris et al. (1988) have made observations of globular and open clusters, using a camera similar to those of the WFPC and a larger number of filters, many of which matched those used in the WFPC. They also derived transformation equations between their magnitudes and the $U B V R I$ magnitudes and colours. Paltoglou \& Bell (1991) and Edvardsson \& Bell (1989) presented large grids of synthetic surface brightness magnitudes for 21 of the WFC pass-bands. The magnitudes were calculated from a set of synthetic spectra for $T_{\text {eff's }}$ in the range 4000 to $7250 \mathrm{~K}$, surface gravities typical of dwarfs and sub-giants, and for metallicities from solar to one thousandth of the solar metallicity. Furthermore, they derived the absolute magnitudes in these 21 pass-bands for a set 
of globular cluster isochrones with different helium abundances, metallicities, oxygen abundances, and ages. The isochrones were taken from VandenBerg \& Bell (1985), Bell \& VandenBerg (1987), Hesser et al. (1987), and McClure et al. (1987). Finally, they presented useful transformations from the 21 WFC pass-bands to various classical photometric systems, like the Johnson $U B V$, Cousins $V R I$, Stromgren ubvy, and Thuan-Gunn uvgr. RoccaVolmerange \& Guiderdoni (1988) derived the integrated magnitudes and colours of model galaxies together with their cosmological evolution at high red-shifts in various UV pass-bands of the HST WFC. Bertola et al. (1995) in order to interpret the UV data for the stars in M 31 acquired with the FOC combined their own observations in the pass-band F150W+F130LP with those of King et al. (1992) in the pass-band F175W and presented synthetic magnitudes and colours that were the preliminary version of the present study. Finally, Yi et al. (1995) presented colour calibrations for the FOC UV filters F140W, F220W and F342W together with isochrones with composition typical of globular clusters.

In this study, first we present large grids of isochrones and integrated magnitudes and colours of single stellar populations (SSP) for several pass-bands of the WFPC2 of the refurbished HST and a few of the old FOC, second with the aid of these results we address the question of galaxy ages. The plan of the paper is as follows.

Section 2 contains the definition of the HST photometric system for the WFPC2 pass-bands F170W (17W), F218W (21W), F300W (30W), F336W (33W), F439W (43W), F450W (45W), F555W (55W), F606W (60W), F702W (70W), F814W (81W), and F850LP (85W), and for the old FOC pass-bands F150W+F130LP (15F), F175W (17F), F220W (22F), and F342W (34F). Thereinafter all pass-bands are indicated either by $\Delta \lambda$ or the abbreviation in brackets.

Section 3 presents the basic colour-temperature relations for the above pass-bands. All magnitudes and colours are obtained using the large library of stellar spectra calculated by Kurucz (1992) however implemented as described by Bressan et al. (1994), Bressan et al. (1996), and Tantalo et al. (1996). The main characteristics of the spectral library in usage are shortly described below. Since we intend that the results of this study can have as wide applicability as possible, independently of our choice for the stellar models to construct the isochrones, we present also tables of conversions as a function of the metallicity, $T_{\text {eff }}$, and gravity that allow to transform the bolometric luminosity of a star into magnitudes of the HST system. The goal is achieved calculating grids of Bolometric Corrections $B C_{\Delta \lambda}^{\mathrm{HST}}$ to be defined below.

Section 4 presents the grids of isochrones in the HST photometry. The ages go from those typical of old globular to those suited to very young clusters and associations. Each isochrone extends from the zero age main sequence (ZAMS) till the end of the asymptotic giant branch (AGB) phase and down to the White Dwarf stage or the core carbon ignition as appropriate for the initial value of the star mass.

Section 5 presents the integrated magnitude and colours of SSPS over the same age range as for the isochrones.

For the sake of brevity, the presentation of the theoretical results in Sects. 4 and 5 is limited to the solar composition $[Y=0.28, Z=0.020]$, even if other compositions are occasionally mentioned. The considered chemical compositions are $[Y=0.23, Z=0.0004],[Y=0.232, Z=0.001]$, $[Y=0.24, Z=0.004],[Y=0.25, Z=0.008],[Y=0.28$, $Z=0.020],[Y=0.352, Z=0.050],[Y=0.475$, $Z=0.10]$. The helium and metal contents vary according to the enrichment law $\Delta Y / \Delta Z=2.5$ (cf. Pagel et al. 1993).

Section 6 presents the CMD of a composite population containing both young and old stars with the aim of illustrating how the morphology of a CMD varies with the pass-bands in use, calling the reader attention on unexpected peculiarities of the CMD in extreme UV passbands.

Section 7 addresses the question of galaxy age and analyzes in some detail the colour-redshift relation of model galaxies in order to check whether suitable observations in the UV can provide useful tools to determine the age of galaxies and/or to discriminate among different models of the Universe, i.e. constrain the parameters $H_{0}$ and $q_{0}$.

Finally, Sect. 8 contains some concluding remarks.

\section{The photometric system of HST}

\subsection{Definition}

According to the core aperture photometry technique (Bushouse 1993), the HST instrumental magnitudes are usually defined as

$m_{\Delta \lambda}=-21.1-2.5 \log \left[(F-B \Sigma) U_{\Delta \lambda} / \epsilon / t\right]$

where $F$ is the total number of counts within the aperture radius, $B$ is the background value within a suitably chosen annulus centered on each star peak, $\Sigma$ is the aperture area, $t$ is the exposure time, $\epsilon$ is the fraction of energy falling into the core aperture with respect to that in the annulus, and $U_{\Delta \lambda}$ is the inverse sensitivity of the instrument mode used (detector + filters), and finally $\Delta \lambda$ stands for the pass-band under consideration. The quantity $(F-B \Sigma) / \epsilon / t$ is the flux of photons per unit time measured by the instrument.

To derive the theoretical counterpart of the above magnitudes, first we calculate from spectral energy distribution of given $\log T_{\text {eff' }}$ 's gravities $(\log g)$ and chemical composition the quantity

$N_{\Delta \lambda}=\int_{\lambda_{\min }}^{\lambda_{\max }}\left(F_{\lambda}(\lambda) /(h c / \lambda)\right) \Gamma_{\Delta \lambda}(\lambda) \mathrm{d} \lambda$ 


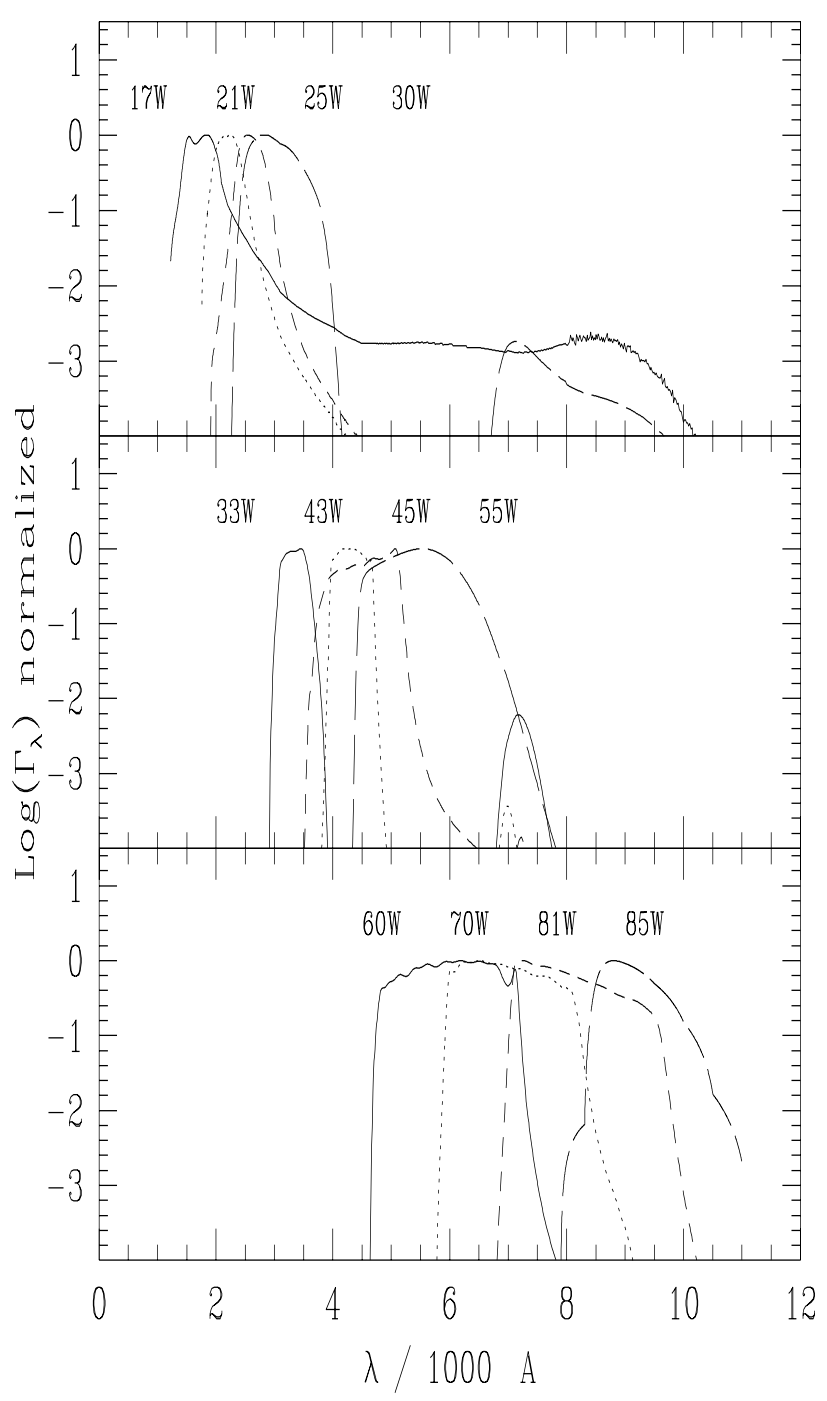

Fig. 1. The functions $\Gamma(\lambda)$ for the indicated pass-bands of the WFPC2 (see the text for more details) The wavelength $\lambda$ is in $\AA$. Each $\Gamma(\lambda)$ is normalized to its peak value. The top Panel shows the filters F170W (solid), F218W (dotted), F255W (dashed), and F300W (long-dashed). The middle panel is for F336W (solid), F439W (dotted), F450W (dashed), F555W (long-dashed). The bottom panel is for F606W (solid), F702W (dotted), F814W (dashed) and F850LP (long-dashed)

where $F_{\lambda}$ is the spectral energy distribution of the source, $\Gamma_{\Delta \lambda}(\lambda)$ is the product of the filter transmission $T_{\Delta \lambda}(\lambda)$ and the response function $R_{\Delta \lambda}(\lambda)$ of the telescope assembly and detector in use, and all other symbols have their usual meaning ( $h$ is the Planck constant and $c$ is the speed of light). According to its definition, $N_{\Delta \lambda}$ is the number of photon emitted per unit of time by an idealsource with $L=L_{\odot}$ and detected by the filter in use. This quantity is then scaled to that received by a collector of unit area when the source is located at the distance of $10 \mathrm{pc}$. Finally,

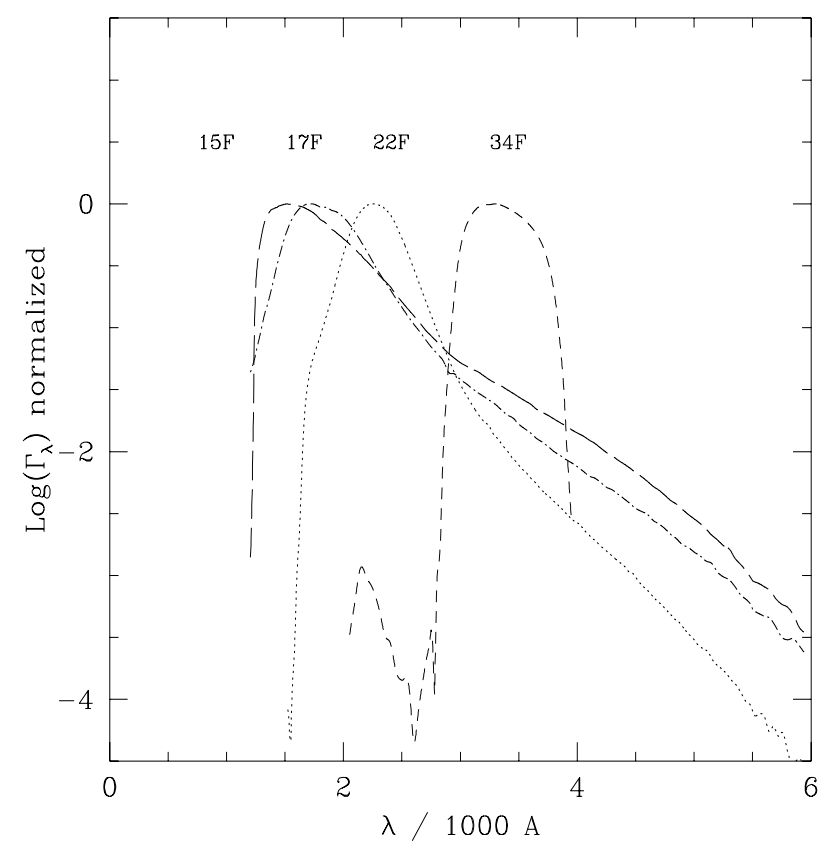

Fig. 2. The functions $\Gamma(\lambda)$ for the indicated pass-bands of the FOC (see the text for more details). The wavelength $\lambda$ is in $\AA$. Each $\Gamma(\lambda)$ is normalized to its peak value. The filters are F150W+F130LP (long dashed), F175W (dotted-dashed), F220W (dotted), and F342W (dashed)

large grids of $N_{\Delta \lambda}$ are computed at varying $T_{\text {eff }}$, gravity, and chemical composition.

Second, we calculate the Bolometric Corrections defined as

$B C_{\Delta \lambda}^{\mathrm{HST}}=M_{\mathrm{bol}, \odot}+21.1+2.5 \log \left[U_{\Delta \lambda} \times \Sigma \times N_{\Delta \lambda}\right]$

where $\Sigma$ is the area of the collecting surface, and $M_{\mathrm{bol}, \odot}$ is the absolute bolometric magnitude of the Sun, for which we adopt 4.72 .

The $B C_{\Delta \lambda}^{\mathrm{HST}}$ are functions of the $T_{\text {eff }}$, gravity, and chemical composition.

Finally, the real absolute magnitude $M_{\Delta \lambda}$ of a star of any luminosity, $T_{\text {eff }}$, gravity, and chemical composition is given by

$M_{\Delta \lambda}=M_{\mathrm{bol}}-B C_{\Delta \lambda}^{\mathrm{HST}}$.

The apparent magnitudes follow from taking into account distance modulus and extinction.

\subsection{Optical characteristics and calibration}

The optical characteristics of the filters in use are given in the WFPC2 and FOC Instrumental Handbooks and do not need to be presented here. Suffice it to recall the main characteristics defining each filter, i.e. the peak wavelength $\lambda_{0}$ and the half-peak width $\Delta \lambda_{1 / 2}$ together with 


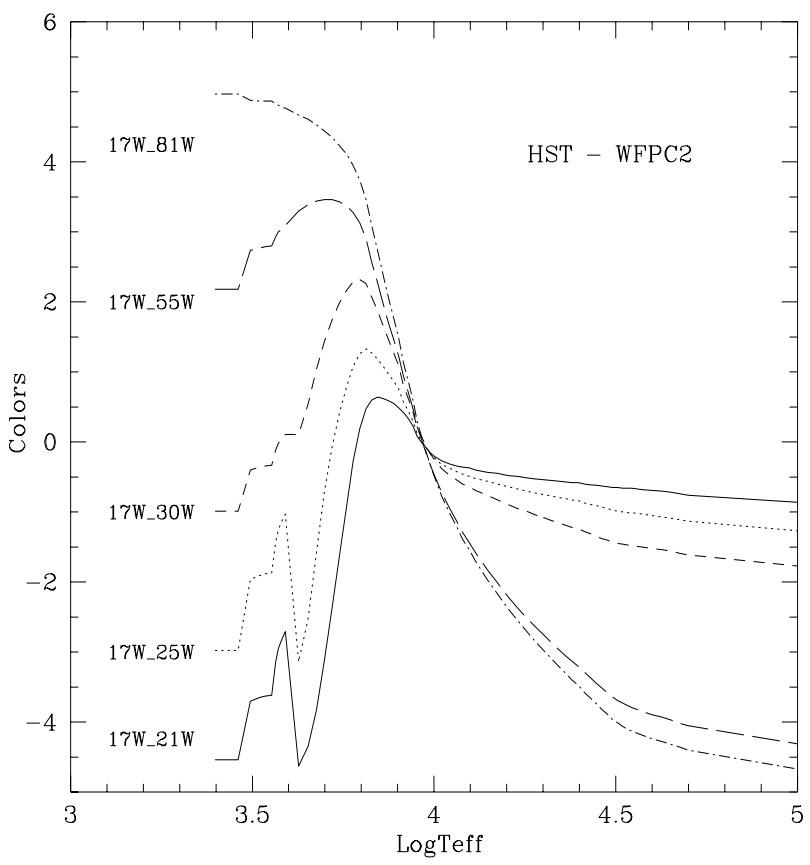

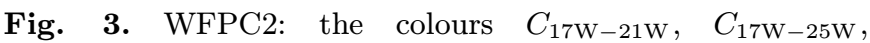
$C_{17 \mathrm{~W}-30 \mathrm{~W}}, C_{17 \mathrm{~W}-55 \mathrm{~W}}$ and $C_{17 \mathrm{~W}-81 \mathrm{~W}}$ versus $\log T_{\text {eff }}$ for the solar composition $[Y=0.28, Z=0.020]$ and $\log g=5$

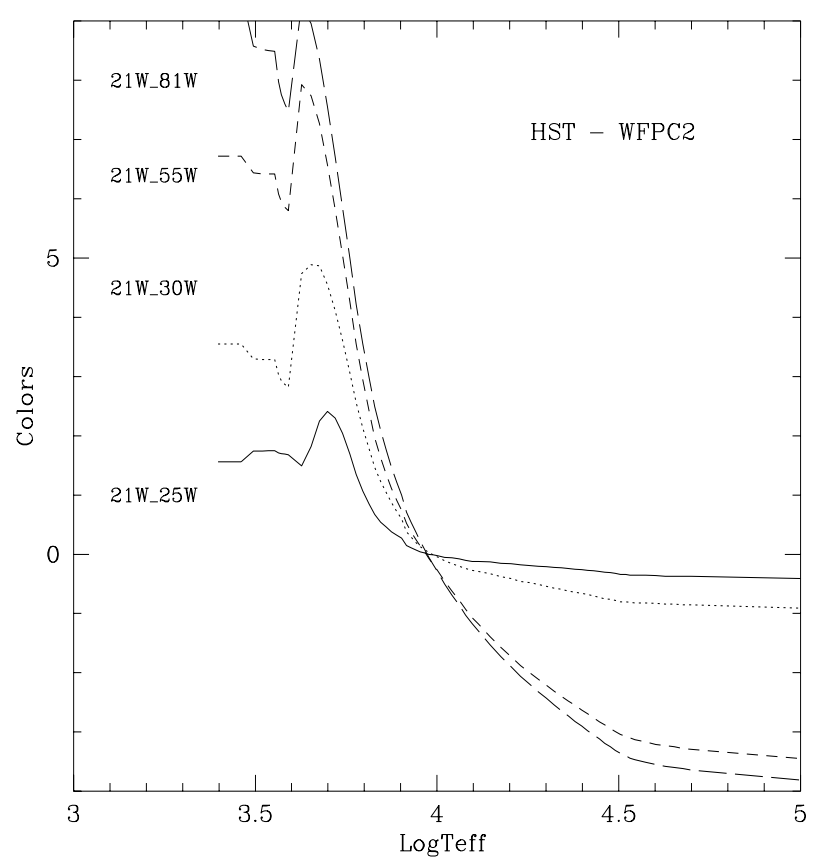

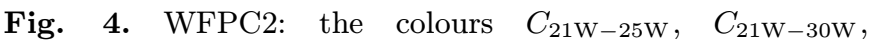
$C_{21 \mathrm{~W}-55 \mathrm{~W}}$, and $C_{21 \mathrm{~W}-81 \mathrm{~W}}$ versus $\log T_{\text {eff }}$ for the solar composition $[Y=0.28, Z=0.020]$ and $\log g=5$

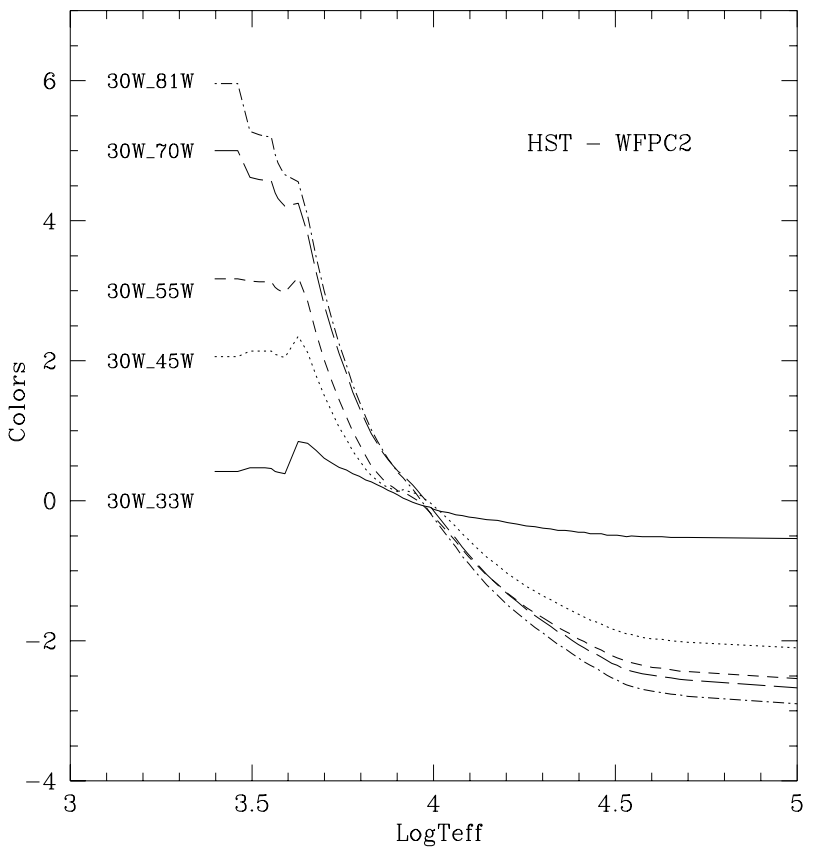

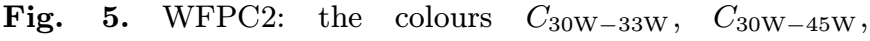
$C_{30 \mathrm{~W}-55 \mathrm{~W}}, C_{30 \mathrm{~W}-70 \mathrm{~W}}$, and $C_{30 \mathrm{~W}-81 \mathrm{~W}}$ versus $\log T_{\text {eff }}$ for the solar composition $[Y=0.28, Z=0.020]$ and $\log g=5$

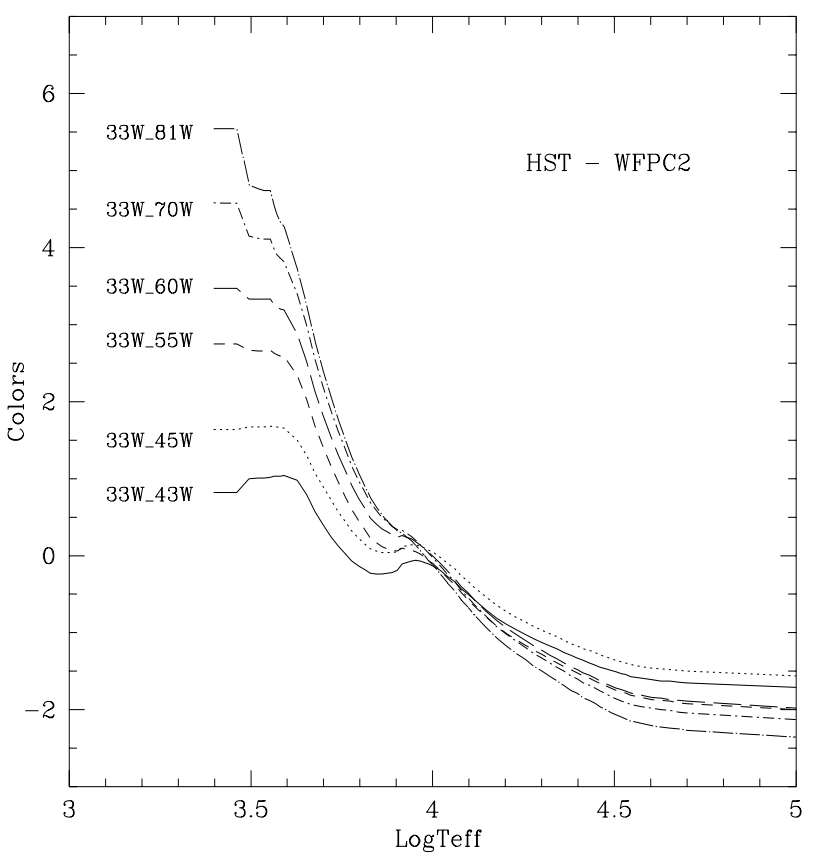

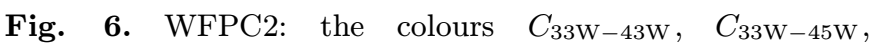

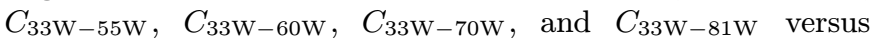
$\log T_{\text {eff }}$ for the solar composition $[Y=0.28, Z=0.020]$ and $\log g=5$ 


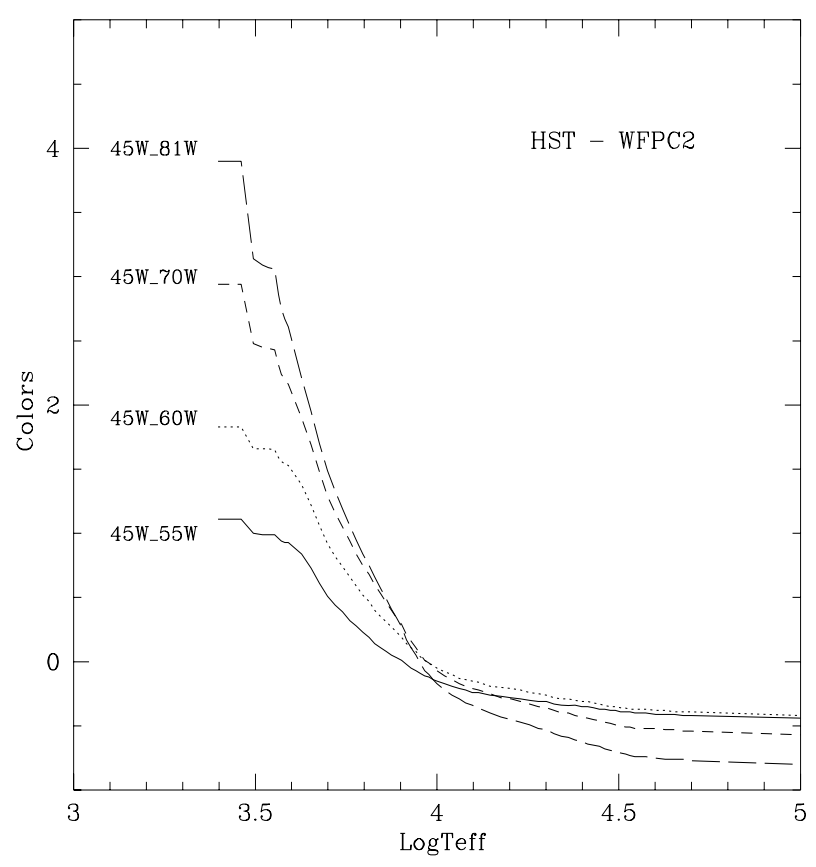

Fig. 7. WFPC2: the colours $C_{45 \mathrm{~W}-55 \mathrm{~W}}, C_{45 \mathrm{~W}-60 \mathrm{~W}}$, $C_{45 \mathrm{~W}-70 \mathrm{~W}}$, and $C_{45 \mathrm{~W}-81 \mathrm{~W}}$ versus $\log T_{\text {eff }}$ for the solar composition $[Y=0.28, Z=0.020]$ and $\log g=5$

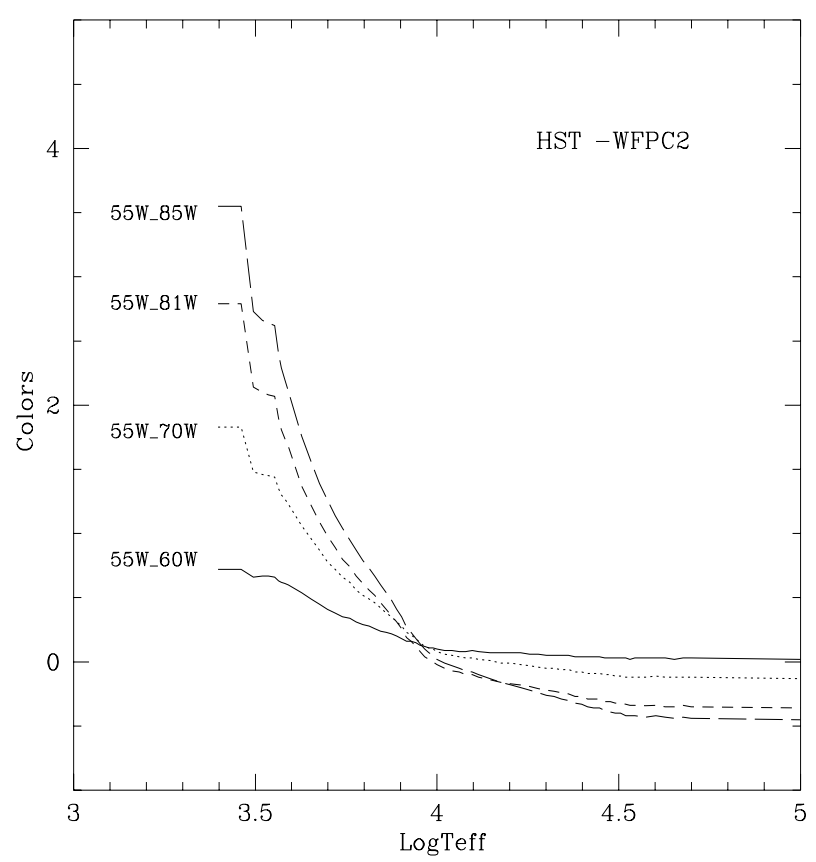

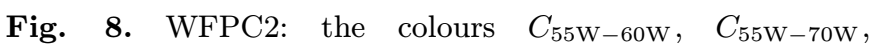
$C_{55 \mathrm{~W}-81 \mathrm{~W}}$, and $C_{55 \mathrm{~W}-85 \mathrm{~W}}$ versus $\log T_{\text {eff }}$ for the solar composition $[Y=0.28, Z=0.020]$ and $\log g=5$

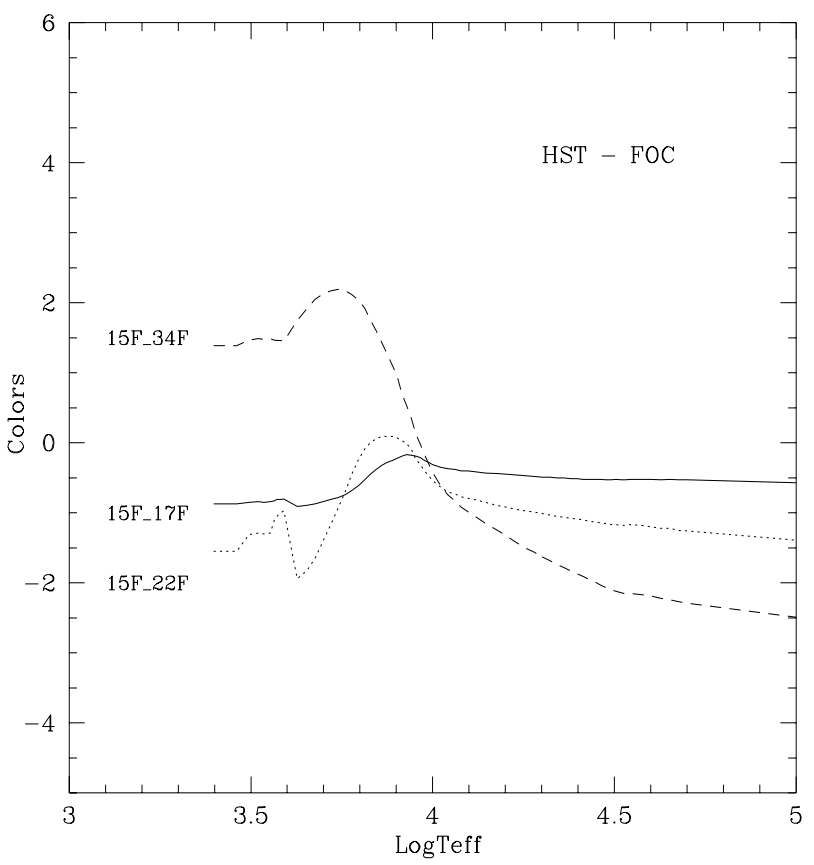

Fig. 9. FOC: the colours $C_{15 \mathrm{~F}-17 \mathrm{~F}}, C_{15 \mathrm{~F}-22 \mathrm{~F}}$ and $C_{15 \mathrm{~F}-34 \mathrm{~F}}$ versus $\log T_{\text {eff }}$ for the solar composition $[Y=0.28, Z=0.020]$ and $\log g=5$

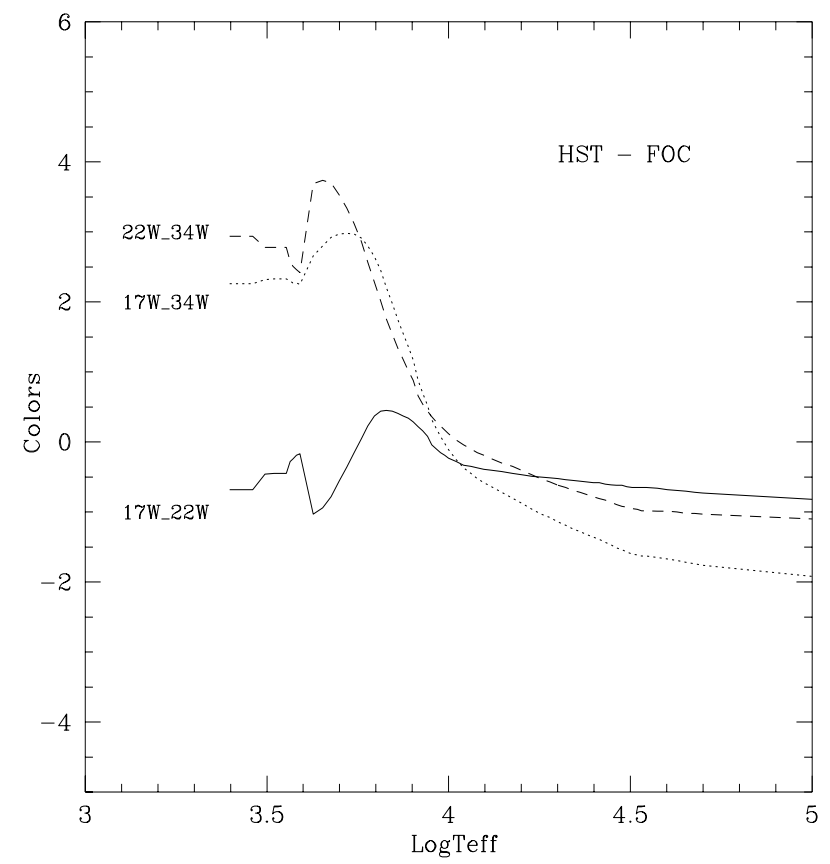

Fig. 10. FOC: the colours $C_{17 \mathrm{~F}-22 \mathrm{~F}}, C_{17 \mathrm{~F}-34 \mathrm{~F}}$ and $C_{22 \mathrm{~F}-34 \mathrm{~F}}$ versus $\log T_{\text {eff }}$ for the solar composition $[Y=0.28, Z=0.020]$ and $\log g=5$ 
Table 1. Main characteristics of the filters in use. All wavelengths are in $\AA$

\begin{tabular}{lccccc}
\hline Filter & Name & $\lambda_{0}$ & $\Delta \lambda_{1 / 2}$ & $\log U_{\Delta \lambda}$ & Note \\
\hline F170W & $17 \mathrm{~W}$ & 1734 & 686 & -15.76 & \\
F218W & $21 \mathrm{~W}$ & 2190 & 407 & -15.94 & \\
F255W & $25 \mathrm{~W}$ & 2587 & 403 & -16.12 & \\
F300W & 30W & 2941 & 757 & -17.06 & Wide $U$ \\
F336W & 33W & 3341 & 439 & -17.04 & $U$-John \\
F439W & $43 \mathrm{~W}$ & 4300 & 476 & -17.64 & $B$-John \\
F450W & $45 \mathrm{~W}$ & 4519 & 957 & -18.02 & Wide $B$ \\
F555W & $55 \mathrm{~W}$ & 5397 & 1226 & -18.31 & $V$-John \\
F606W & $60 \mathrm{~W}$ & 5934 & 1498 & -18.41 & Wide $V$ \\
F702W & $70 \mathrm{~W}$ & 6862 & 1378 & -18.23 & Wide $R$ \\
F814W & $81 \mathrm{~W}$ & 7924 & 1497 & -17.95 & $I$-John \\
F850LP & $85 \mathrm{~W}$ & 9065 & 983 & -17.23 & \\
\hline \multicolumn{5}{c}{ FOC } \\
F150WL & $15 \mathrm{~F}$ & 1360 & 298 & -16.07 & \\
F175W & $17 \mathrm{~F}$ & 1730 & 716 & -16.11 & \\
F220W & $22 \mathrm{~F}$ & 2260 & 470 & -16.67 & \\
F342W & $34 \mathrm{~F}$ & 3410 & 702 & -15.74 & \\
\hline
\end{tabular}

the inverse sensitivity $U_{\Delta \lambda}$ of the instrument mode used (detector + filters). These parameters are summarized in Table 1.

The constants $U_{\Delta \lambda}$ for the WFPC2 pass-bands are derived from the calibrated WFPC2 synthetic photometric system of Holtzman et al. (1995a,b) where the zero points refer to Vega. These constants are calculated for the gain factor $14 \mathrm{e}^{-} / \mathrm{DN}$ and aperture radius of $0.5^{\prime \prime}$. Wherever possible the correction to the aperture of $3^{\prime \prime}$ has been applied using the data contained in Table $5 \mathrm{~B}$ of Holtzman et al. (1995a,b) for the WF3. This has been feasible for the pass-bands $17 \mathrm{~W}, 21 \mathrm{~W}, 25 \mathrm{~W}, 30 \mathrm{~W}, 33 \mathrm{~W}, 43 \mathrm{~W}, 45 \mathrm{~W}, 55 \mathrm{~W}$ and $81 \mathrm{~W}$. The magnitudes for the remaining pass-bands $(45 \mathrm{~W}, 60 \mathrm{~W}, 70 \mathrm{~W}$, and $85 \mathrm{~W})$ refer to the aperture of $0.5^{\prime \prime}$ radius.

In the case of the FOC pass-bands, the constants $U_{\Delta \lambda}$ are taken directly from the Instrumental Handbook.

The functions $\Gamma_{\Delta \lambda}(\lambda)$ for the WFCP2 are shown in Fig. 1 whereas those for the FOC are given in Fig. 2. The functions $\Gamma_{\Delta \lambda}(\lambda)$ s are displayed in Figs. 1 and 2 normalized to their peak value. Notice in Fig. 1 that all the filters short-ward of $45 \mathrm{~W}$ have long tails toward the visible/red wavelength region, some of them showing secondary peaks. This anomalous behavior of the filters will immediately reflect into a non monotonic behavior of the colours (see below).

\subsection{The spectral library}

The library of stellar spectra in use is the one developed by Bressan et al. (1994) and Tantalo et al. (1996). The main body of the spectral library is from Kurucz (1992) however extended in the high and low temperatures ranges. For $T_{\text {eff }}>50000 \mathrm{~K}$ pure black-body spectra is assigned. For $T_{\text {eff }}<3500 \mathrm{~K}$, the new catalog of stellar fluxes by Fluks et al. (1994) together with their scale of $T_{\text {eff }}$ is adopted. All other details can be found in the studies quoted above.

\section{General properties of the photometric systems}

The dependence of the $B C_{\Delta \lambda}^{\mathrm{HST}}$ on the $T_{\text {eff }}$ (at given gravity and chemical composition) governs the appearance of a star in the CMD. This is best shown by the colour- $T_{\text {eff }}$ relation for any pair of pass-bands. The colour is simply given by

$C_{\Delta \lambda-\Delta \lambda^{\prime}}=M_{\Delta \lambda}-M_{\Delta \lambda^{\prime}}$

with $\Delta \lambda$ and $\Delta \lambda^{\prime}=17 \mathrm{~W}, 21 \mathrm{~W}, 25 \mathrm{~W}, 30 \mathrm{~W}, 33 \mathrm{~W}, 43 \mathrm{~W}$, $45 \mathrm{~W}, 55 \mathrm{~W}, 60 \mathrm{~W}, 70 \mathrm{~W}, 81 \mathrm{~W}, 85 \mathrm{LW}$ for the WFPC; $\Delta \lambda$ and $\Delta \lambda^{\prime}=15 \mathrm{~F}, 17 \mathrm{~F}, 22 \mathrm{~F}, 33 \mathrm{~F}$ for the FOC, with obvious meaning of the symbols. In all cases $\Delta \lambda<\Delta \lambda^{\prime}$.

The relations for each group are shown in the series of Figs. 3 through 10 limited to the case of solar composition and gravity typical of main sequence stars $(\log g=5)$. It is apparent that colours involving one or two UV passbands do not possess a monotonic relation with $T_{\text {eff }}$. With the standard Johnson system, it is natural to see colours becoming redder as $T_{\text {eff }}$ decrease, as long as the colours are defined as bluer magnitudes minus redder magnitudes. With the HST system the colour becomes bluer again when the temperature is cooler than a certain value that depends on the pass-band (hereafter the colour turnover). The turnover temperature somewhat depends on the gravity and chemical composition because the spectral energy distribution varies with them. Finally, the colour turnover does no longer occur for the visible-red pass-bands.

The turnover is caused by the combination of two effects. First the UV pass-bands are affected by the the socalled visible/red-leak problem, i.e. long tails of significant transmittance at wavelengths much longer than the nominal peak value (cf. Figs. 1 and 2). For very hot stars, the amount of flux gathered in the visible/red-leak region is small and the effect of this on the colour is negligible. As the star gets cooler the amount of flux falling into the visible/red-leak region becomes more and more important and so its effect on the colour. The other effect is that the various UV filters have visible/red-leaks that intersect each other. For instance the visible/red-leak of the filter $15 \mathrm{~W}$ is higher than the visible/red-leak of the filters $21 \mathrm{~W}$ and $25 \mathrm{~W}$, whereas the visible/red-leak of the filter F30W is higher than the previous ones. This anomalous behaviour tends to disappear going to filters of longer and longer peak wavelength. Analogous considerations hold for the UV filters of the FOC.

The obvious consequence of the colour turnover is that one cannot determine a unique value of the temperature from the colours at least as long as some UV bands are involved. Although the combination of a UV pass-band with one, whose peak is at much longer wavelength can improve upon this point of ambiguity, there are cases in which the effect is still there (see for instance the case of $C_{15 \mathrm{~W}-81 \mathrm{~W}}$ or $C_{17 \mathrm{~W}-81 \mathrm{~W})}$. 


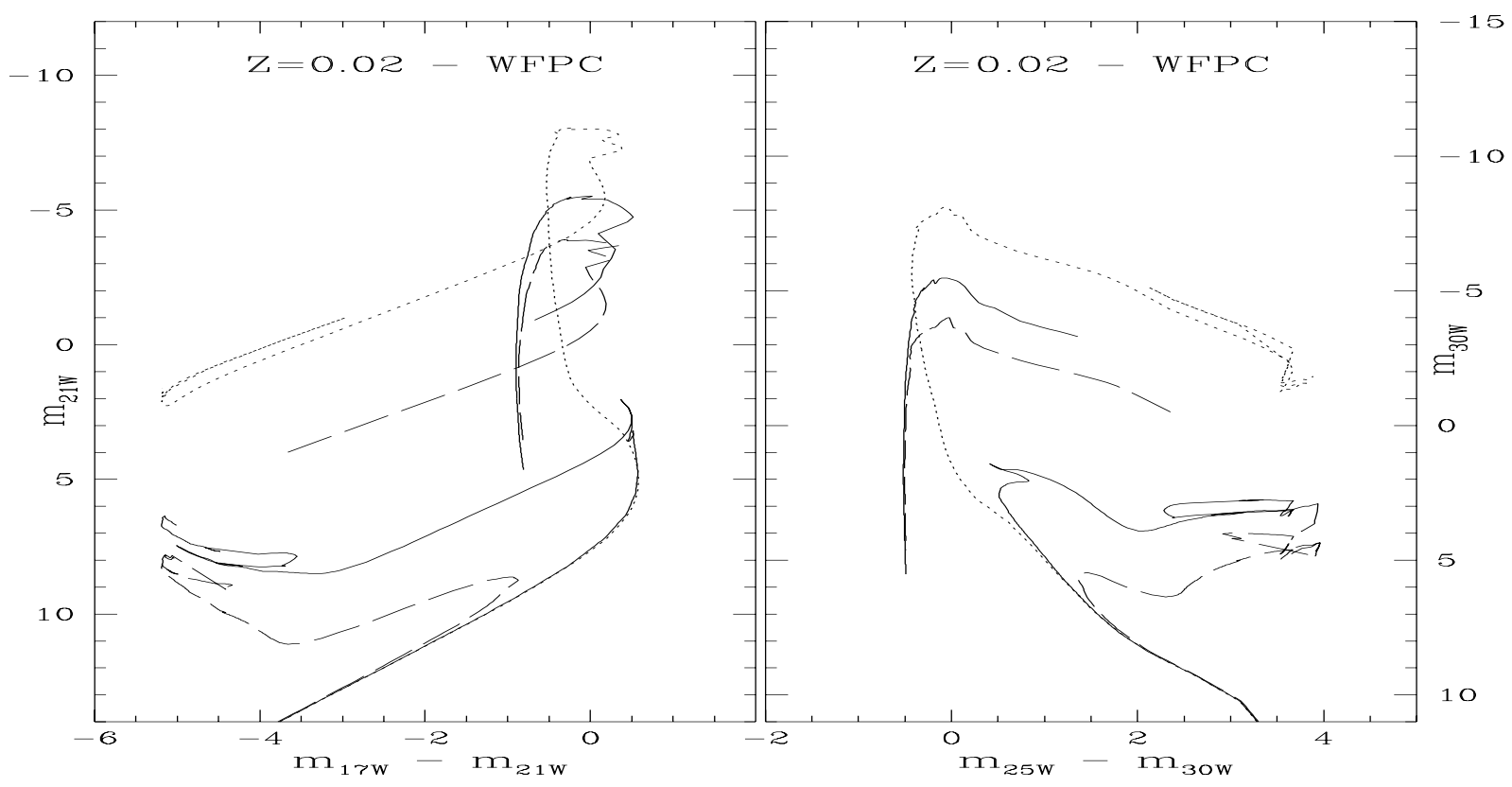

Fig. 11. Left Panel: three isochrones in the CMD for the WFPC pass-bands $17 \mathrm{~W}$ and $21 \mathrm{~W}$. Dashed line, solid line and dotted line are for the 10 Gyr, 1 Gyr and 0.01 Gyr ages, respectively. Right Panel: the same but for the pass-bands $25 \mathrm{~W}$ and $30 \mathrm{~W}$

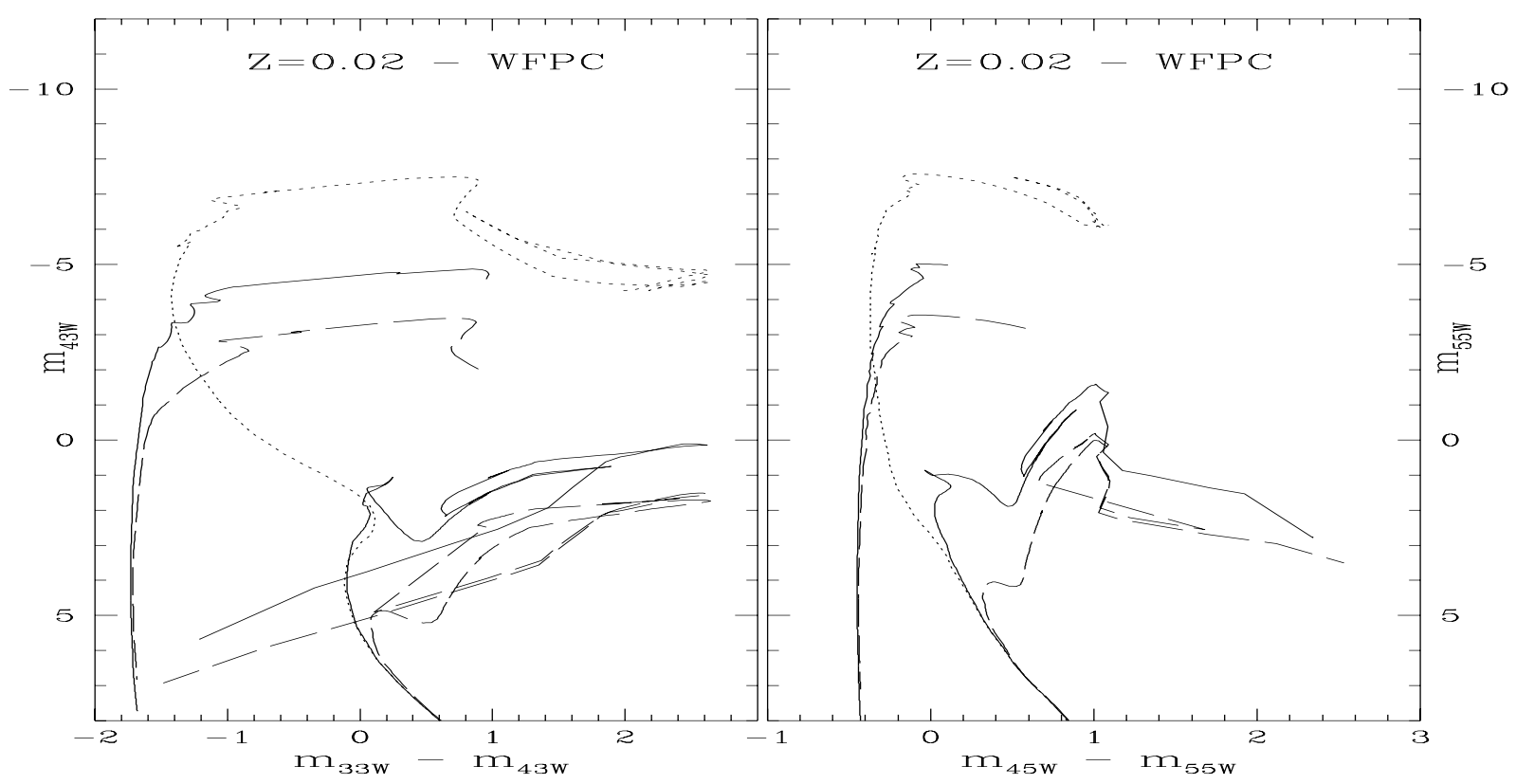

Fig. 12. Left Panel: three isochrones in the CMD for the WFPC pass-bands $33 \mathrm{~W}$ and $43 \mathrm{~W}$. Dashed line, solid line and dotted are for the $10 \mathrm{Gyr}, 1 \mathrm{Gyr}$ and $0.01 \mathrm{Gyr}$ ages, respectively. Right Panel: the same but for the pass-bands $45 \mathrm{~W}$ and 55W 


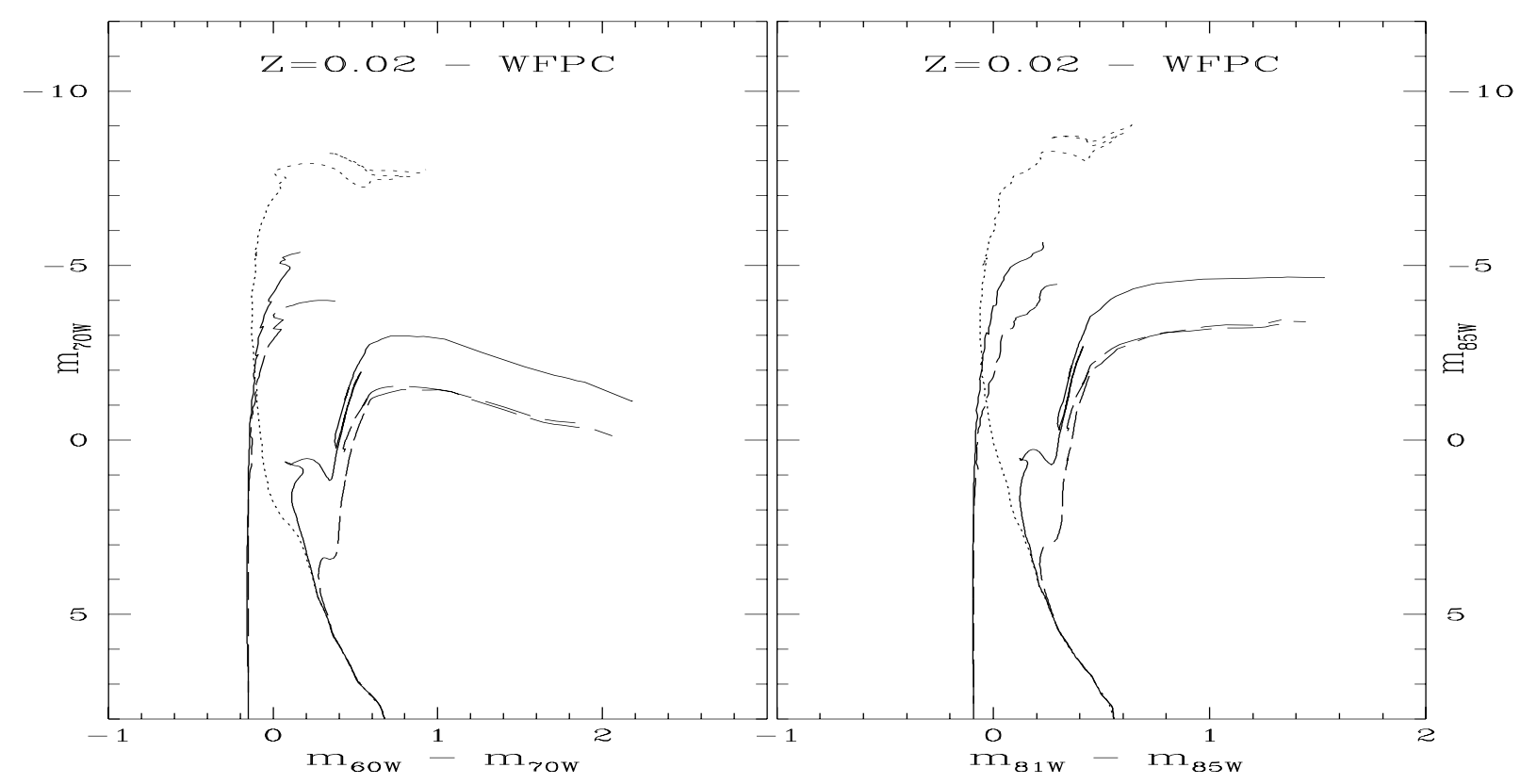

Fig. 13. Left Panel: three isochrones in the CMD for the WFPC pass-bands $60 \mathrm{~W}$ and $70 \mathrm{~W}$. Dashed line, solid line and dotted line are for the $10 \mathrm{Gyr}, 1 \mathrm{Gyr}$ and $0.01 \mathrm{Gyr}$ ages, respectively. Right Panel: the same but for the pass-bands $81 \mathrm{~W}$ and $85 \mathrm{~W}$

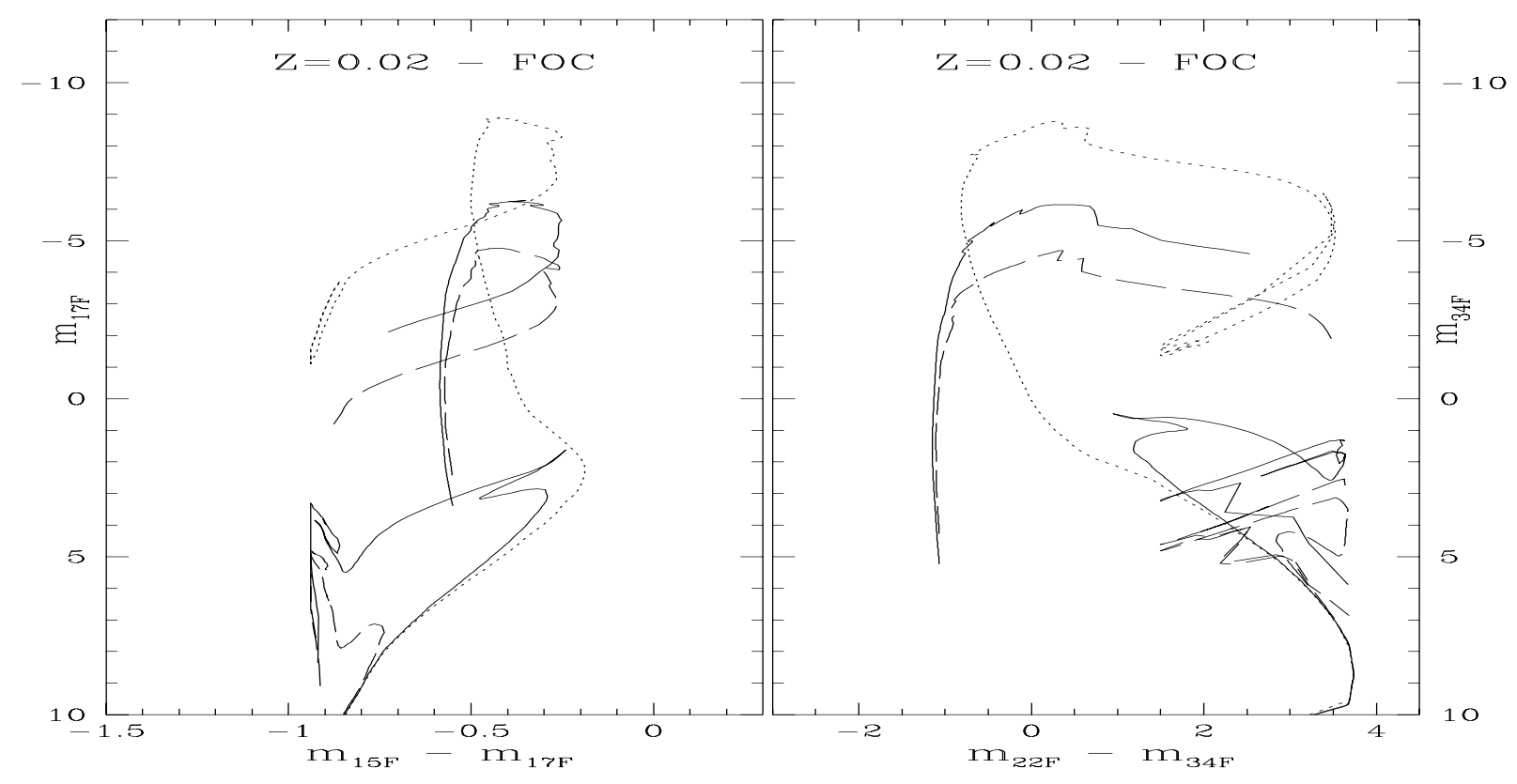

Fig. 14. Left Panel: three isochrones in the CMD for the FOC pass-bands $15 \mathrm{~F}$ and $17 \mathrm{~F}$. Dashed line, solid line and dotted line are for the 10 Gyr, 1 Gyr and 0.01 Gyr ages, respectively. Right Panel: the same but for the pass-bands $22 \mathrm{~F}$ and $34 \mathrm{~F}$ 


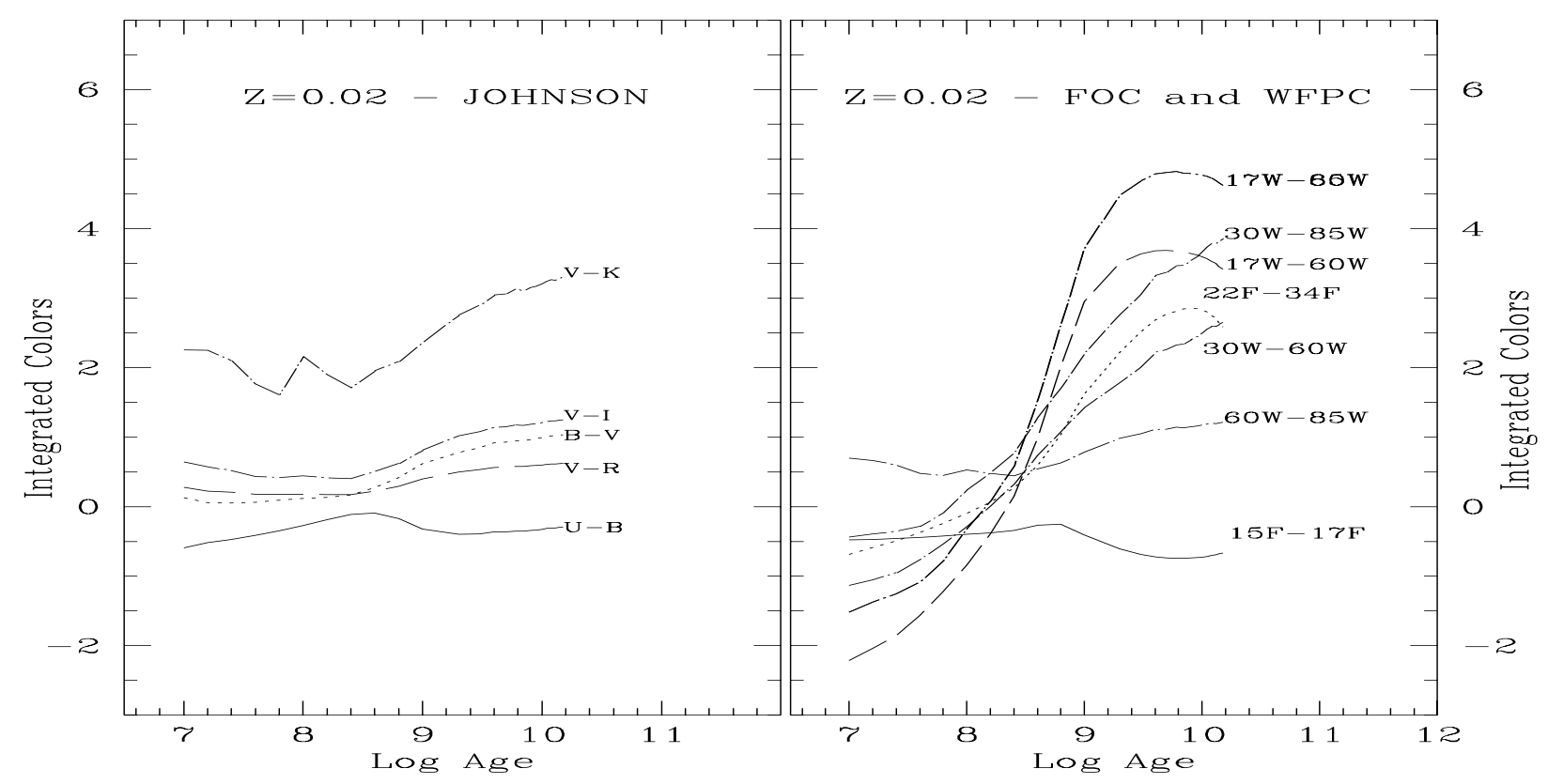

Fig. 15. Integrated colours as function of the age for the SSP with solar composition $[Y=0.28, Z=0.02]$ and the Salpeter initial mass function

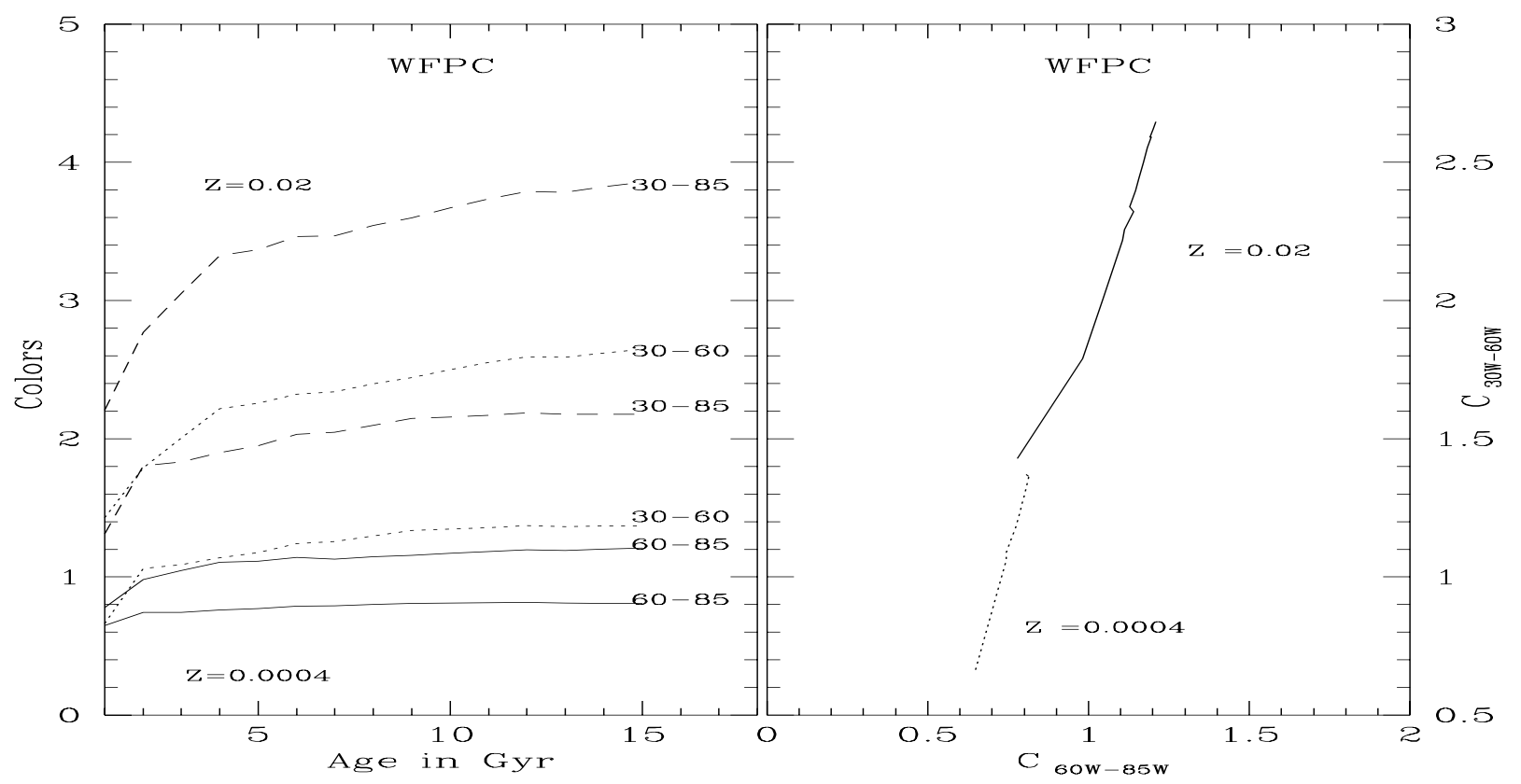

Fig. 16. Integrated colours as function of the age for the SSP with solar composition $[Y=0.28, Z=0.02]$ and the Salpeter initial mass function. Left Panel: $C_{30 \mathrm{w}-60 \mathrm{w}}$ (dotted), $C_{30 \mathrm{w}-85 \mathrm{w}}$ (dashed), and $C_{60 \mathrm{w}-85 \mathrm{w}}$ (solid). For each colour the lower curve refers to $Z=0.0004$, the upper curve to $\mathrm{Z}=0.02$. Right panel: two colour plane $\mathrm{C}_{30 \mathrm{~W}-60 \mathrm{~W}}$ versus $C_{60 \mathrm{~W}-85 \mathrm{~W}}$ 
Table 2. Model Galaxies: $\tau, t_{\mathrm{gw}}$ and Age are in Gyr, the galaxy mass is in $10^{12} M_{\odot}$

\begin{tabular}{lcccccccccccc}
\hline Model & Mass & $\tau$ & $\nu$ & $H_{0}$ & $q_{0}$ & $t_{\mathrm{gw}}$ & Age & $\langle Z\rangle$ & $Z_{\max }$ & $(B-V)$ & $(V-K)$ & $(1550-\mathrm{V})$ \\
\hline & & & & & & & & & & & & \\
$\mathrm{A}$ & 3 & 0.1 & 15 & 50 & 0 & 0.15 & 16.42 & 0.027 & 0.049 & 1.00 & 3.29 & 3.69 \\
$\mathrm{~B}$ & 3 & 0.1 & 3 & 50 & 0 & 0.87 & 16.42 & 0.039 & 0.097 & 0.94 & 3.47 & 1.24 \\
$\mathrm{C}$ & 3 & 10 & 0.5 & 50 & 0 & & 16.42 & 0.063 & 0.097 & 0.38 & 3.89 & -1.32 \\
\hline & & & & & & & & & & & & \\
$\mathrm{A}$ & 3 & 0.1 & 15 & 50 & 0.5 & 0.15 & 12.26 & 0.027 & 0.049 & 0.99 & 3.35 & 4.62 \\
$\mathrm{~B}$ & 3 & 0.1 & 3 & 50 & 0.5 & 0.87 & 12.26 & 0.039 & 0.097 & 0.92 & 3.44 & 1.32 \\
\hline & & & & & & & & & & & & \\
$\mathrm{A}$ & 3 & 0.1 & 15 & 80 & 0 & 0.15 & 10.38 & 0.027 & 0.049 & 0.98 & 3.40 & 4.80 \\
$\mathrm{~B}$ & 3 & 0.1 & 3 & 80 & 0 & 0.87 & 10.38 & 0.039 & 0.097 & 0.90 & 3.47 & 1.22 \\
\hline & & & & & & & & & & & & \\
$\mathrm{A}$ & 3 & 0.1 & 15 & 80 & 0.5 & 0.15 & 7.66 & 0.027 & 0.049 & 0.95 & 3.39 & 5.23 \\
$\mathrm{~B}$ & 3 & 0.1 & 3 & 80 & 0.5 & 0.87 & 7.66 & 0.039 & 0.097 & 0.86 & 3.46 & 2.08 \\
\hline
\end{tabular}

The effect of the colour turnover on the observed CMD is easy to foresee and is discussed in the next section by means of select isochrones.

Finally, we notice that colours like $C_{17 \mathrm{~W}-\Delta \lambda^{\prime}}$, $C_{21 \mathrm{~W}-\Delta \lambda^{\prime}}, \quad C_{30 \mathrm{~W}-\Delta \lambda^{\prime}}$, and $C_{33 \mathrm{~W}-\Delta \lambda^{\prime}}$ with $\Delta \lambda^{\prime}>$ $17 \mathrm{~W}, 21 \mathrm{~W}, 30 \mathrm{~W}, 33 \mathrm{~W}$, respectively, show pronounced dips at $\log T_{\text {eff }} \simeq 3.5$ which are caused by the secondary peaks in the functions $\Gamma_{\Delta \lambda}(\lambda)$ pointed out in the previous sections.

There is a final remark to be made. The colour- $\log T_{\text {eff }}$ relations for the WFPC2 system obey the basic condition that at $\log T_{\text {eff }}=4.0$ all the colours are zero or nearly zero (see Figs. 3 through 8), which is typical of the ground based photometric systems (Johnson for instance). This means that the calibration of the $U_{\Delta \lambda}$ has been properly made. In the case of the FOC system, the above condition is no longer always satisfied, cf. Figs. 9 and 10. At $\log T_{\text {eff }}=4.0$ the colours may be significantly different from zero. Similar behaviour is also visible in the FOC colours calculated by Yi et al. (1995). This implies that the corresponding $U_{\Delta \lambda}$ requires further calibration. Although this point can be easily fixed up by adding suitable shifts to the magnitudes or suitably correcting the $U_{\Delta \lambda}$, we prefer to present results for the original $U_{\Delta \lambda}$.

\section{Isochrones in the HST photometry}

Perhaps the most extended library of isochrones in literature is by Bertelli et al. (1994). The great merit of this library is that it satisfies the basic criteria of adequacy and accuracy of the input physics in the stellar model calculations, and homogeneity. The isochrones span the age range from a few $10^{6}$ yr to beyond $18 \mathrm{Gyr}$, and cover all evolutionary phases from the zero age main sequence up to either the stage of PN formation and cooling toward the White Dwarf regime or central C-ignition depending upon the mass of the most evolved star in the isochrone.
The chemical compositions are in the range $[Z=0.0001$, $Y=0.23]$ to $[Z=0.1, Y=0.475]$ and obey the enrichment law $\Delta Y / \Delta Z=2.5$. For the purposes of this study, the Bertelli et al. (1994) isochromes have been revised in relation to the algorithm adopted to describe mass loss by stellar wind during the AGB phase. In this paper, following Marigo et al. (1996) we make use of a semi-empirical formalism which relates the mass loss rate $\dot{M}$ to the pulsational period $\mathrm{P}$ of Mira variables and pulsating $\mathrm{OH} / \mathrm{IR}$ stars both in the Galaxy and in the LMC (Vassiliadis \& Wood 1993). The advantage of this prescription is the onset of the superwind phase which develops naturally on the AGB, instead of the ad hoc sudden increase that is needed with classical Reimers-like laws, cf. Reimers (1975) and Renzini \& Voli (1981). No other details of the isochrone construction are given here for the sake of brevity.

Figures 11-13 show a few selected CMDs for the WFPC2 pass-bands whereas Fig. 14 is the same but for the FOC. Each CMD contains three isochrones with age 10 Gyr, 1 Gyr and 0.01 Gyr and composition $[Y=0.28$, $Z=0.020]$.

All the CMDs are constructed using the conventional format so that the left side has a smaller colour value.

But for the cases with the extreme UV pass-bands such as $15 \mathrm{~F}, 17 \mathrm{~F}, 22 \mathrm{~F}, 43 \mathrm{~F}, 17 \mathrm{~W}, 21 \mathrm{~W}, 30 \mathrm{~W}$, and $33 \mathrm{~W}$, the CMDs look normal and (as expected) much like to those in the classical Johnson system. In contrast, in the case of the far UV pass-bands the above mentioned colour turnover occurs, giving rise to unfamiliar sequences in CMDs. This implies that care must be paid when UV data for stars and star clusters are to be interpreted and associated to a certain evolutionary phase. See for instance the CMD of 47Tuc studied by Paresce et al. (1991).

It is worth noticing that, depending on the particular colour in usage, if the CMD looks normal at old ages it may reverse itself at young ages and viceversa. Furthermore there are CMDs in which particular evolutionary 
stages are enhanced with respect to others. For instance in the CMD for the pass-bands $30 \mathrm{~W}$ and $85 \mathrm{~W}$, the turnoff is always brighter than or as bright as the remaining evolutionary stages. Magnitudes and colours in these passbands are potentially good age indicators.

There are CMDs in which young isochrones are fully degenerate in the sense that all evolutionary stages beyond the main sequence are first pushed to much fainter magnitudes and second to colours that are indistinguishable from the main sequence phase.

\section{Integrated colours of SSP}

In Fig. 15 we present integrated colors as a function of the age for the SSP with solar composition $[Y=0.28$, $Z=0.020]$. The left panel shows some colours of the classical Johnson system, whereas the right panel shows some colours of the HST-WFCP2 and HST-FOC systems. For these latter the colours on display are $C_{17 \mathrm{~W}-60 \mathrm{~W}}$, $C_{17 \mathrm{~W}-85 \mathrm{~W}}, C_{30 \mathrm{~W}-60 \mathrm{~W}}, C_{30 \mathrm{~W}-85 \mathrm{~W}}, C_{60 \mathrm{~W}-85 \mathrm{~W}}, C_{15 \mathrm{~F}-17 \mathrm{~F}}$,

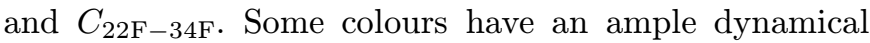
range at varying age and metallicity. For instance, for ages older than 1 Gyr the colours $C_{30 \mathrm{~W}-60 \mathrm{~W}}$ and $C_{30 \mathrm{~W}-85 \mathrm{~W}}$ have a linear age dependence with amplitude of about 3 mag. The dependence on metallicity is best shown by the left panel of Fig. 16 in which the evolution of the colours $C_{30 \mathrm{~W}-60 \mathrm{w}}, C_{30 \mathrm{~W}-85 \mathrm{w}}$ and $C_{60 \mathrm{~W}-85 \mathrm{~W}}$ for the SSPs with $Z=0.0004$ and $Z=0.02$ is displayed. At given age, the dynamical range of these colours is about $3.5 \mathrm{mag}$. In the two color plane $C_{60 \mathrm{~W}-85 \mathrm{~W}}$ versus $C_{30 \mathrm{~W}-60 \mathrm{~W}}$ shown in the right panel of Fig. 16 the SSPs of different metallicity are located on a unique almost linear relation along which both the age and the metallicity vary. Even if the well known age-metallicity degeneracy is still there, the above colours are useful to confine ages and metallicity.

An interesting point to be noticed is that all colours but the extreme blue ones like $(U-B)$ and $C_{15 \mathrm{~F}-17 \mathrm{~F}}$ show evidence of the onset in the SSP of the AGB stars at the age of about 0.1 Gyr that make them significantly redder. The effect is not the same in all colours, being more pronounced in $(V-K)$ or $C_{17 \mathrm{~W}-85 \mathrm{~W}}, C_{30 \mathrm{~W}-85 \mathrm{~W}}$ and similar.

It is worth reminding the reader that the magnitudes of these SSP refer to the Salpeter (1955) initial mass function $\Phi(M)=C M^{-2.35}$ (in number) with the normalization constant $C=1$. To be applied to real stellar populations (for instance clusters of assigned total mass and/or number of stars) these magnitudes must be shifted by the quantity

$-2.5 \log C$

where $C$ is the real normalization constant (see Chiosi et al. 1988, 1989; Bertelli et al. 1994 for details). It goes without saying that these data cannot be used to model the integrated magnitudes and colours of a SSP with a different initial mass function. In such a case new isochrones and integrated quantities must be calculated. Nevertheless, despite the limitations in the initial mass function, these results give a realistic idea of how the integrated properties of SSP vary with time and metallicity (cf. Girardi et al. 1995).

\section{The CMD of a composite population: NGC 205}

For the purpose of illustration we present here the CMD of a composite stellar system containing both very old and young stars, such as for instance an old galaxy with some recent star formation. This example is meant to mimic the stellar content of NGC 205 in which in addition to the old component young stars are observed. Since the early studies by Baade (1951), this galaxy has long been considered to show evidence of ongoing star formation because of the UV-bright stars as well as large number of regions of absorption and dust (Hodge 1973). Mould et al. (1983, 1984) determined a metallicity $[M / H]=-1.2 \pm 0.2$ from the CMD in the outer regions. Peletier (1993) studied the color profile of the galaxy and found a very blue center and colors that get redder slowly going outward. Because of the presence of blue luminous stars in the center, the color gradient is interpreted as a gradient in age. The existence of luminous blue stars sets a limit to the age of the recent episode of star formation at a few $10^{6}$ yr. Furthermore, the galaxy contains a number of bright red stars, which are so bright that they either are red supergiants or evolved AGB stars (Gallagher \& Mould 1981). Following Peletier (1992), NGC 205 can be modeled as an old galaxy with some young stars in the center. The age of the underlying old component is more difficult to assess. The study of Mould et al. $(1983,1984)$ perhaps implies an age not younger than a few Gyr. Using the static population synthesis technique, Bica et al. (1990) inferred from the observed spectral energy the existence of an old component whose metallicity lies the range $-2 \leq Z / Z_{\odot} \leq-1$, and whose age is older than $5 \mathrm{Gyr}$ with typical value of $12-13$ Gyr. They also confirmed the existence of a young component with age in the range 0.01 Gyr to a few 0.1 Gyr, which contributes to about $80 \%$ of the visible light. With the aid of the Bertelli et al. (1994) isochrones and luminosity functions, we estimate that the young component though dominating the visible light yet contains only about $2 \%$ of the total mass of stars in the galaxy.

On the basis of these observational hints, we model the star formation history in NGC 205 as a burst-like episode at constant rate whose duration is from $0.5 \mathrm{Gyr}$ ago to the present time, say a few $0.001 \mathrm{Gyr}$, superposed to an exponential law that was maximum in the past, say 13 Gyr ago, and then quickly declined toward the present. The rate of decline of the exponential component and the amplitude of star formation in the burst with respect to that in the previous phase, are chosen in such a way that the above percentages in the relative total mass of stars born in the two episodes are matched. We find that an 

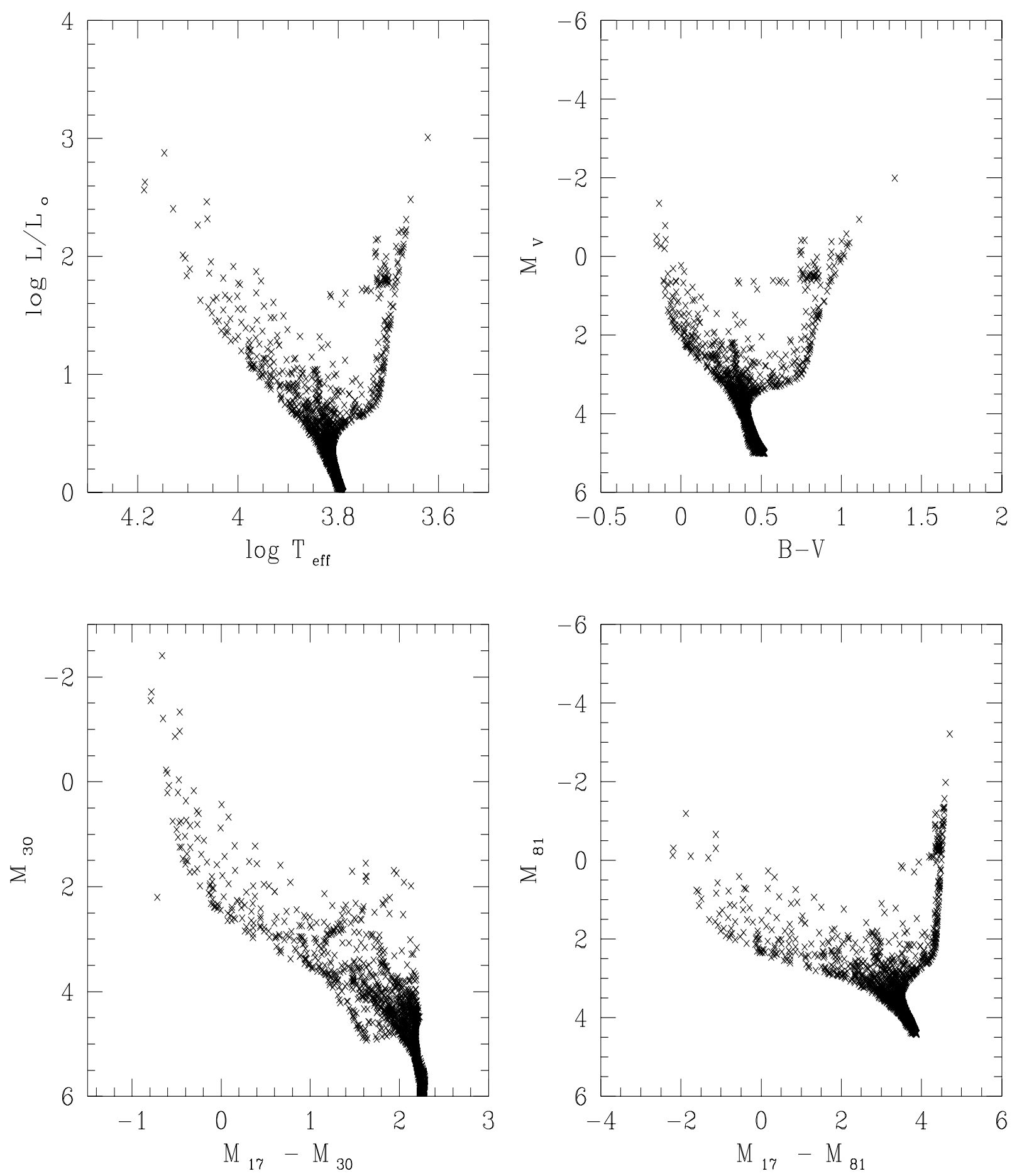

Fig. 17. Synthetic CMDs for an assembly of stars whose star formation history is meant to resemble the one that took place in the nearby galaxy NGC 205. The various panels show the same CMD but in different planes. Notice how the RGB and HB

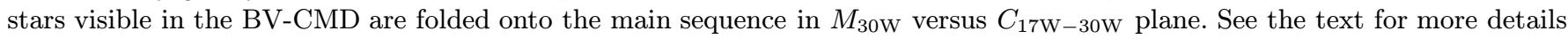


e-folding of $1 \mathrm{Gyr}$ and relative amplitude of the burst of about 0.1 are good choices.

Figure 17 shows four synthetic CMDs: the theoretical plane, the classical $(B-V)$ versus $M_{V}$ plane, the $C_{17 \mathrm{~W}-30 \mathrm{~W}}$ versus $M_{30 \mathrm{~W}}$ plane, and finally the $C_{17 \mathrm{~W}-81 \mathrm{~W}}$ versus $M_{81 \mathrm{~W}}$ plane. The distortion of the CMD induced by the non monotonic behaviour of the colour $C_{17 \mathrm{~W}-30 \mathrm{~W}}$ is evident. Indeed the RGB, HB and AGB sequences are folded onto the main sequence so that disentangling the various components is very difficult if not impossible. Similar behaviours are expected with other UV pass-bands such as the $17 \mathrm{~W}, 21 \mathrm{~W}, 22 \mathrm{~F}$ and $34 \mathrm{~F}$. This is a remarkable feature to be kept in mind when analyzing the data acquired with the UV pass-bands of HST. The CMDs in the other pass-bands look normal.

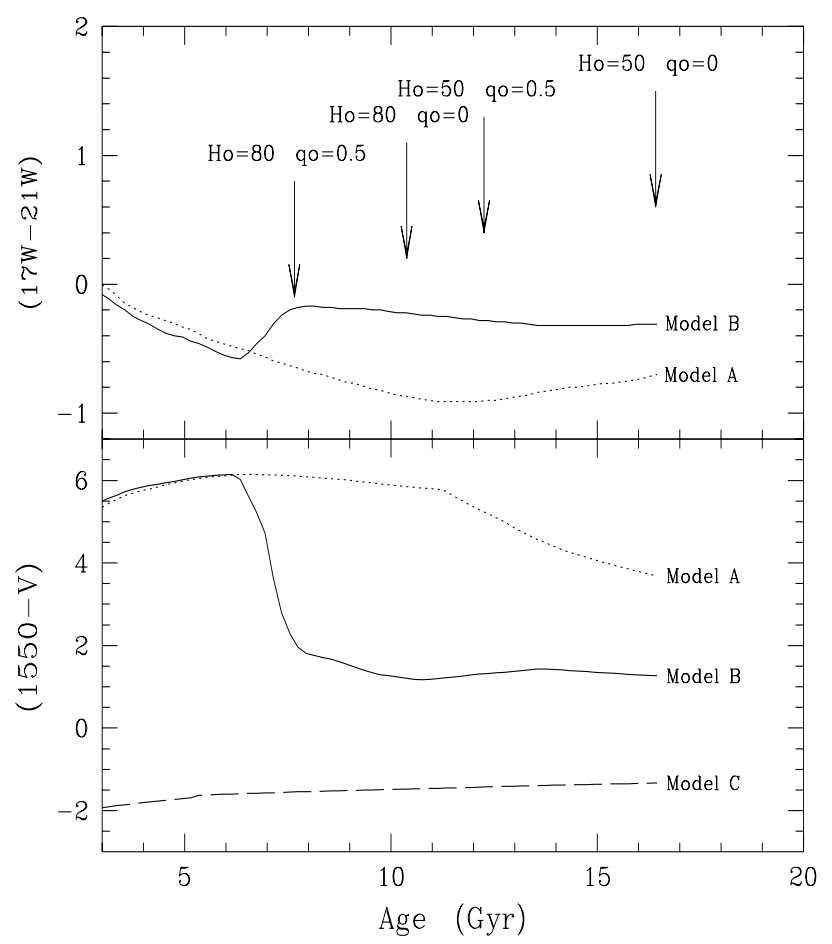

Fig. 18. The colour evolution of Models A, B and C as a function of the rest-frame age. The three models have different histories of star formation and chemical enrichment. See the text for more details. Upper panel: the colour $C_{17 \mathrm{~W}-21 \mathrm{~W}}$ The vertical arrows show the maximum age of the galaxy for different choices of the cosmological parameters $H_{0}$ and $q_{0}$ and $z_{\text {for }}=5$ (see also the entries of Table 2). Lower panel; the same but for the colour (1550-V)

\section{UV magnitudes and colours of galaxies}

In this section we address the long debated question whether the colour evolution of galaxies exhibits signatures that can be used as age indicators. Renzini \&
Buzzoni (1986) first suggested that the variation in broadband colours such as $V-K$ expected to occur in a SSP at the onset of the first AGB and RGB stars at the ages $t\left(M_{\mathrm{UP}}\right) \simeq 0.1$ Gyr and $t\left(M_{\mathrm{HeF}}\right) \simeq 1 \mathrm{Gyr}$, could be used as an age indicator for those galaxies closely resembling SSP, i.e. elliptical galaxies, if trace of this variation named Phase Transitions can be detected at suitable values of the red-shift. The analysis of this problem by Bressan et al. (1994) and Tantalo et al. (1996), first clarified that in a SSP only the variation caused by AGB stars at the age of about 0.1 Gyr can be detected whereas that of the RGB stars is masked by the presence of the former.

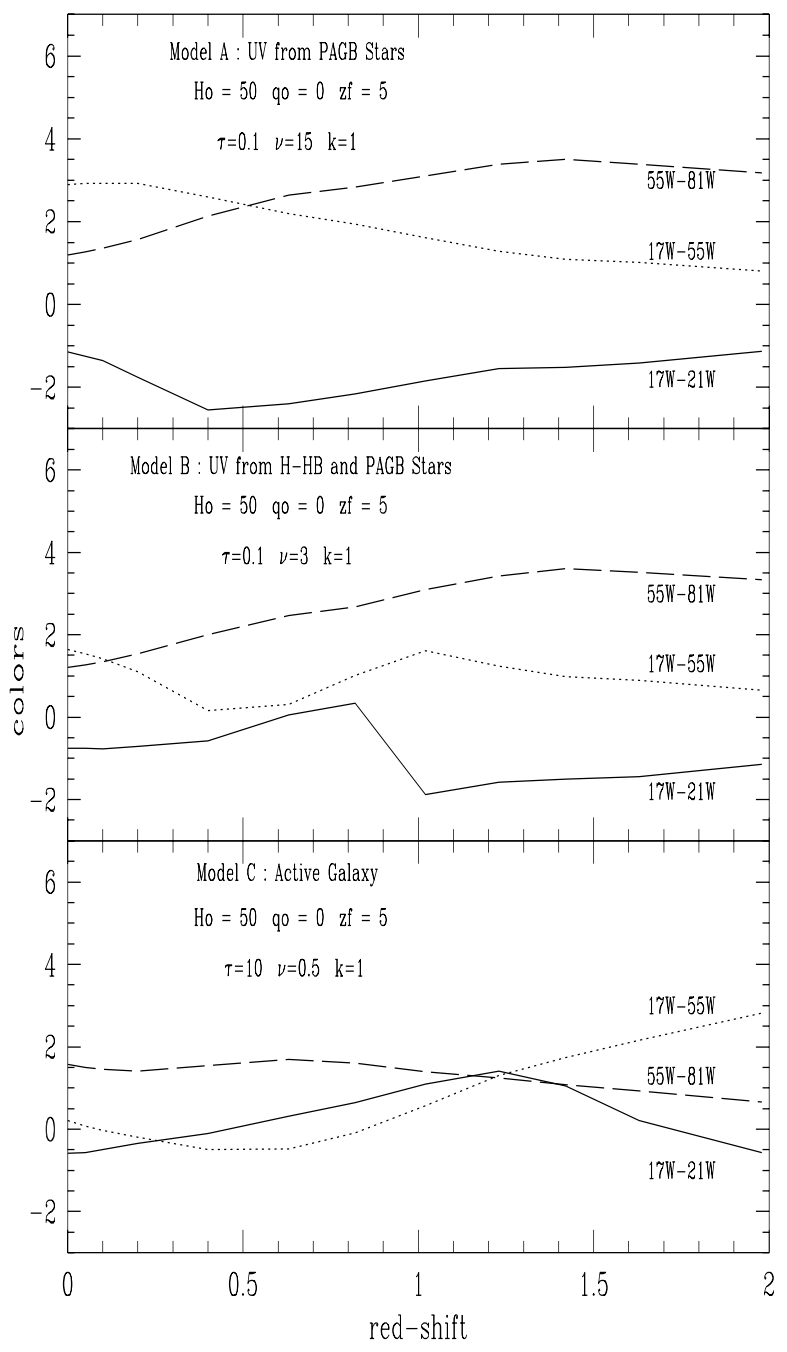

Fig. 19. UV colours of Model A (top panel), Model B (central panel) and Model $\mathrm{C}$ (lower panel) as a function of the red-shift. For purpose of comparison the classical colour $(V-I)$,

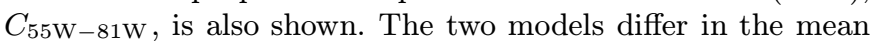
and maximum metallicity. See the text for more details 
The explanation of this resides in the Fuel Consumption Theorem of Renzini \& Buzzoni (1986). Applied to an elliptical galaxy conceived as an assembly of stars undergoing passive evolution since the initial prominent phase of star formation, the extremely young age of the epoch at which the AGB colour variation occurs, implies large values of the red-shift. The analysis of this problem by Bressan et al. (1994) and Tantalo et al. (1996) has shown that this signature in the rest-frame $(V-K)$ cannot be traced back in the colour corresponding to the red-shift implied by the age of 0.1 Gyr, at least for any reasonable combination of the three parameters defining a cosmological model of the Universe, i.e. the Hubble constant $H_{0}$, the deceleration parameter $q_{0}$, and finally the red-shift of galaxy formation $z_{\text {for }}$. Indeed the cosmological distortion of the spectrum wipes out the colour signature. The quest is to look for a feature occurring in a recent past so that small values of the red-shift and little cosmological distortions are involved. Standard broad-band colours such as $(B-V),(V-R), \ldots(V-K)$ are not useful to this purpose because going back in time either they vary smoothly or even remain constant over several Gyr.

Bressan et al. (1994) and Tantalo et al. (1996) have suggested that the uprise in the UV flux at the onset of the H-HB and AGB manque stars can be used as a probe of galaxy ages. Since these stars are expected to appear at the rest-frame age of about 5.6 Gyr for $\Delta Y / \Delta Z=2.5$, a sudden change in the colour $(1550-\mathrm{V})$ should be observable at relatively low red-shifts, perhaps reachable with the present day space instrumentation. The precise value of the red-shift corresponding to the above age depends on the particular model of the Universe in usage.

Aim of this section is to quantify the Bressan et al. (1994) and Tantalo et al. (1996) predictions on the ability of UV colours to probe the age of galaxies. For the sake of clarity first we summarize the key properties of the UV emission detected in elliptical galaxies and the type of stars currently indicated as responsible of this phenomenon, and then make detailed predictions for the colour-red-shift evolution of these stellar systems.

\subsection{UV excess in elliptical galaxies: The data}

The basic information on UV emission in elliptical galaxies (Burstein et al. 1988) can be summarized as follows:

(1) All studied elliptical galaxies have detectable UV flux short-ward of about $2000 \AA$.

(2) There are large galaxy to galaxy differences in the level of the UV flux. The intensity of the UV emission is measured by the colour $(1550-\mathrm{V})$.

(3) The colour (1550-V) correlates with the index Mg2, the velocity dispersion $\Sigma$, and the luminosity (mass) of the galaxy. The few galaxies (e.g. NGC 205) in which active star formation is seen do not obey these relations.

(4) Another important constraint is posed by the HUT observations by Ferguson et al. (1991) and Ferguson \&
Davidsen (1993) of the UV excess in the bulge of M 31. In this galaxy the UV emission shows a drop-off shortward of about $1000 \AA$ whose interpretation requires that the temperature of the emitting source must be about $25000 \mathrm{~K}$. Only a small percentage of the $912 \leq \lambda$ $\leq 1200 \AA$ flux can be coming from stars hotter than $30000 \mathrm{~K}$ and cooler than $20000 \mathrm{~K}$. See also Brown et al. (1995) for more recent data.

\subsection{UV Excess in elliptical galaxies: The sources}

Excluding ongoing star formation, the UV excess owes its origin to an old component that gets hot enough to power the integrated spectral energy distribution (ISED) of a galaxy in the far UV regions. Four possible candidates are envisaged (cf. Greggio \& Renzini 1990; Bressan et al. 1994; and Tantalo et al. 1996). The appearance of the various types of UV sources is governed by several important physical factors, each of which is affected by a certain degree of uncertainty still far from being fully assessed. These are the efficiency of mass loss during the RGB and AGB phases, the enrichment law $\Delta Y / \Delta Z$, and finally for the specific case of the P-AGB stars the detailed relation between the initial and final mass of the stars at the end of the AGB phase.

(1) The classical post asymptotic giant branch (PAGB) stars (see Bruzual 1992; Bruzual \& Charlot 1993; Charlot \& Bruzual 1991), which are always present in the stellar mix of a galaxy. However, they cannot be the sole source of UV flux because of their high $T_{\text {eff }}$ (about $100000 \mathrm{~K}$ ) and lack of sufficient fuel (cf. Greggio \& Renzini 1990). Another point of uncertainty is the precise relation between the P-AGB mass and the turn-off mass (and hence age), which is far from being established. The Weidemann (1987) relation provides the most favorable case for being P-AGB stars an important source of UV flux. However, the response of the UV flux to details of this relation (for instance its dependence on the metallicity) is so strong that firm conclusions cannot yet be reached (Bressan 1996). We remind the reader that for ages older than about $10 \mathrm{Gyr}$, the whole problem is driven by the initial-final mass relation in the mass range 0.8 to $1.0 M_{\odot}$. Finally, it is worth mentioning that P-AGB stars have perhaps been detected with HST observations in the nucleus of M 31 (Bertola et al. 1995) where they seem to contribute by as much as $50 \%$ to the UV light.

(2) Very blue HB (VB-HB) stars of extremely low metallicity (Lee 1994). These stars have $T_{\text {eff }}$ hotter than about $15000 \mathrm{~K}$ but much cooler than those of the P-AGB stars. Therefore, depending on their actual $T_{\text {eff }}$, they can generate ISEDs in agreement with the observational data. In addition to a marginal difficulty with the age, which turns out to be older than commonly assigned to globular clusters (13 \pm 2 Gyr, Fusi-Pecci \& Cacciari 1991), there is the question whether their luminosity and relative frequency are compatible with the observed ISED of elliptical 


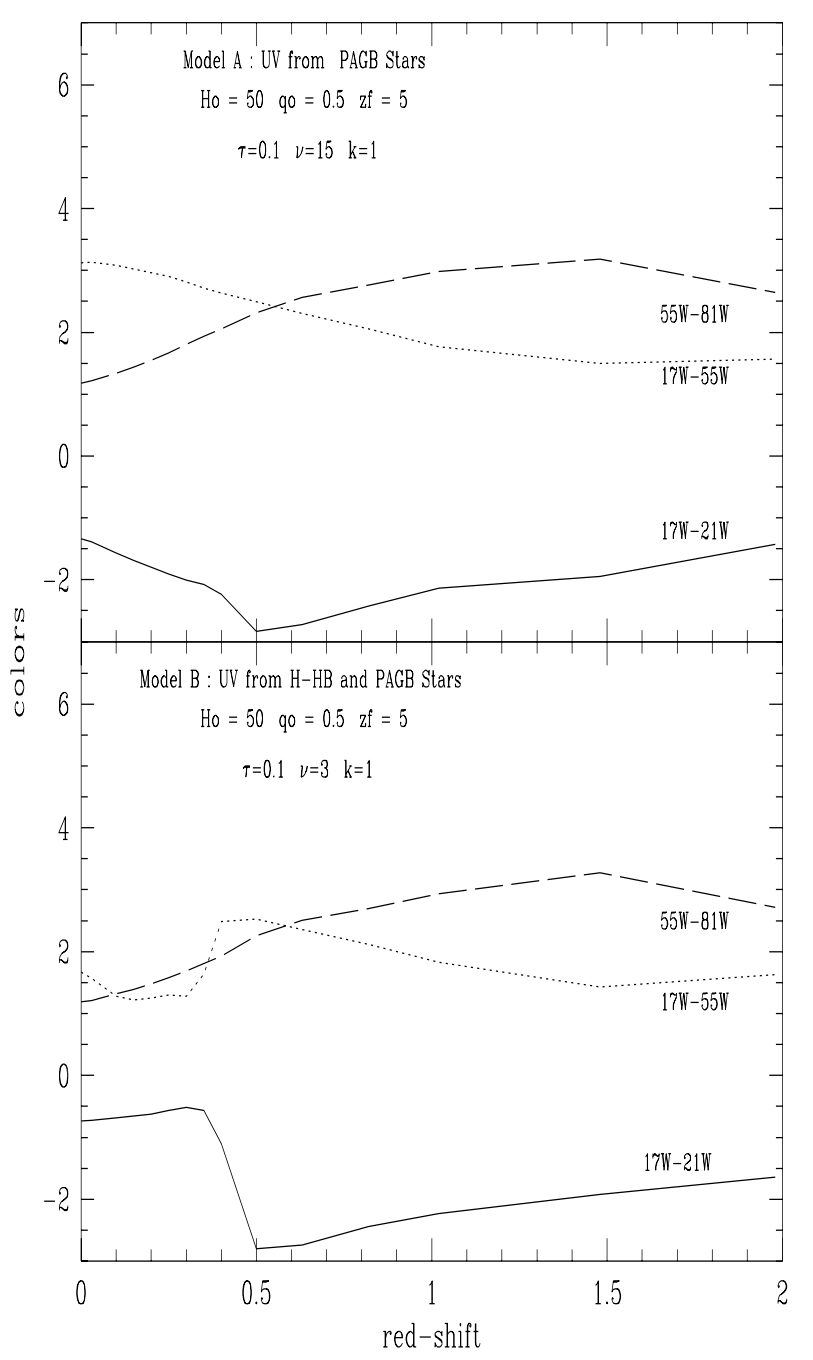

Fig. 20. UV colours of Models A (top panel) and B (bottom panel) as a function of the red-shift. The cosmological parameters are $H_{0}=50 \mathrm{~km} \mathrm{~s}^{-1} \mathrm{Mpc}^{-1}, q_{0}=0.5$. For purpose of comparison the classical colour $(V-I), C_{55 \mathrm{~W}-81 \mathrm{~W}}$, is also shown. The two models differ in the kind of star formation. See the text for more details

galaxies in the range $2000<\lambda 3500 \AA$. Indeed, Bressan et al. (1994) and Tantalo et al. (1996) pointed out that the observed ISEDs hint that only very few stars with metallicity lower than about $Z=0.008$ ought to exist in a typical elliptical galaxy (the analog of the G-Dwarf Problem in the solar vicinity).

(3) The H-HB and AGB-manque stars of high metallicity (say $Z>0.07$ ) which are expected to be present albeit in small percentages in the stellar content of bulges and elliptical galaxies in general (cf. Bressan et al. 1994; Tantalo et al. 1996). Indeed, these stars have $T_{\text {eff }}$ in the right interval and generate ISEDs whose intensity drops short-ward of about $1000 \AA$ by the amount indicated by the observational data. With normal mass loss and $\Delta Y / \Delta Z$, the first H-HB and AGB manque stars occur at the age of about $5.6 \mathrm{Gyr}$. This age is lowered if $\Delta Y / \Delta Z$ is higher than 2.5 and mass loss during the RGB phase is enhanced with respect to the value given by the classical Reimers (1975) law (cf. Dorman et al. 1993, 1995).

(4) Finally, the analog of the above H-HB and AGBmanqué stars, but generated by enhancing the mass loss rate during the RGB phase at increasing metallicity. These types of stars have been named by Dorman et al. (1993, 1995) extremely hot HB objects (E-HB). They share nearly the same properties of the H-HB and AGB-manqué stars. The main difficulty with this option is the uncertainty concerning the metallicity dependence of the mass loss rate during the RGB phase (cf. Carraro et al. 1996).

\subsection{Models of elliptical galaxies}

Bressan et al. (1994, 1996) and Tantalo et al. (1996) elaborated new chemo - spectro - photometric models of elliptical galaxies particularly designed to match the colourmagnitude relation (CMR), cf. Bower et al. (1992), and to provide a robust explanation for the UV flux and its dependence on the galactic luminosity (and hence mass), the index $\mathrm{Mg}_{2}$, and the velocity dispersion $\Sigma$. No details of these models are given here for the sake of brevity. Suffice it to recall that Bressan et al. (1994) made use of the closed-box approximation, whereas Tantalo et al. (1996) adopted the infall description. In both cases the enrichment law is $\Delta Y / \Delta Z=2.5$ and the mass-loss rates for the RGB phase, are the Reimers (1975) law with $\eta=0.35$ in Bressan et al. (1994) and $\eta=0.45$ in Tantalo et al. (1996). Finally, the models allow for galactic winds halting star formation. Galactic winds are at the base of the current interpretation of the CMR for elliptical galaxies (Bower et al. 1992). The models used in the analysis below are from Tantalo et al. (1996) with gas accretion time scale $\tau=0.1$ Gyr and rate of star formation $\Psi(t)$ proportional to the current value of the gas mass: $\Psi(t)=\nu M_{\mathrm{g}}(t)^{k}$ with $k=1$ and $\nu$ an efficiency parameter.

\subsection{Can the UV colours probe the galaxy ages?}

Given these premises, we consider three galactic models characterized by the parameters $\tau$, and $\nu$. The main properties of these models are summarized in Table 2, which contains the galaxy mass, the mass accretion time scale, the star formation efficiency, the adopted cosmological parameters $H_{0}$ and $q_{0}$, the age at the onset of galactic winds $t_{\mathrm{gw}}$, the total age of the galaxy, the mean and maximum metallicity in stars, and the present age colours $(B-V)$ and $(V-K)$ of the Johnson-Cousins system and the standard $(1550-\mathrm{V})$. The various groups of models have the same physical properties but differ in the age because of 


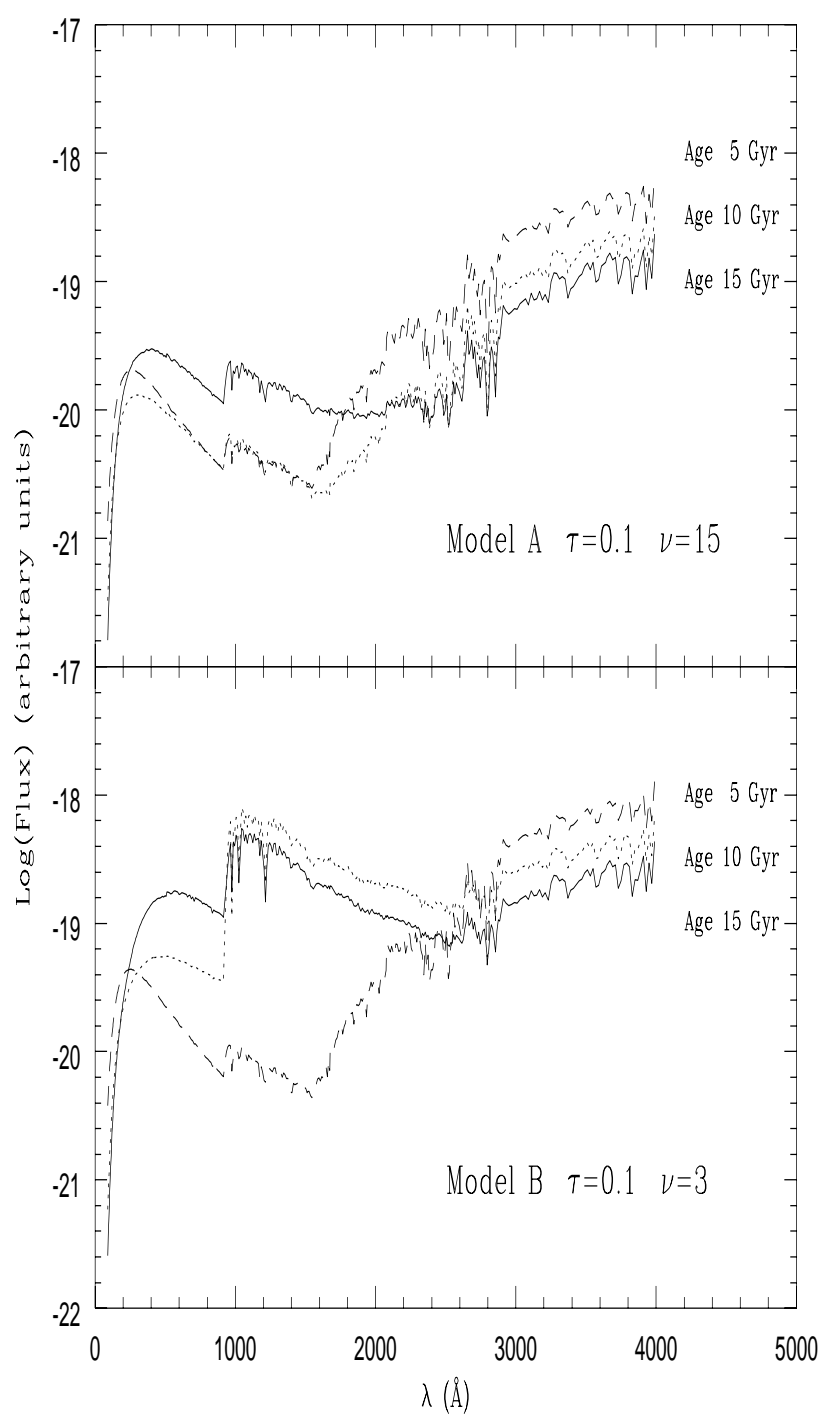

Fig. 21. The ISED of Models A and B for three different values of the age, i.e. 5 (long dashed), 10 (dotted) and 15 Gyr (solid)

the different choice for $H_{0}$ and $q_{0}$. For all the models, the red-shift of galaxy formation is assumed to be $z_{\text {for }}=5$.

The models under consideration are meant to represent three extreme cases: Model A with $\tau=0.1$ Gyr and $\nu=15$ undergoes the wind phase halting any further star formation activity in a very early past. The metallicity cannot grow to the threshold value required to activate the UV emission by H-HB and AGB-manqué stars and only PAGB stars are present. Model B suffers from galactic wind at later ages. The maximum metallicity goes above the threshold value to activate the H-HB and AGB-manqué channels (the fraction of stars in these metallicity bins is however very small). Now P-AGB, H-HB and AGBmanqué stars all concur to generate the UV flux. Finally, Model C has very long mass accretion time scale $(\tau=10$

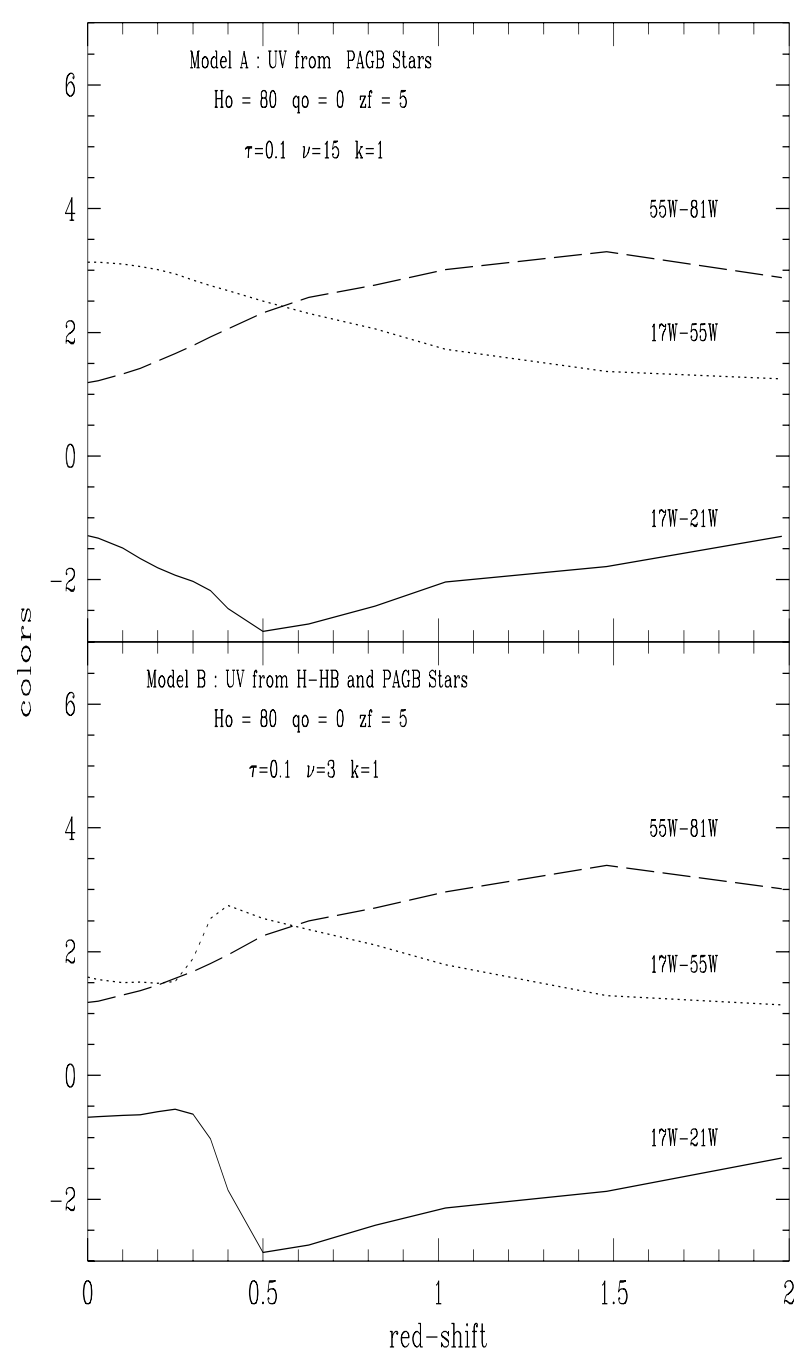

Fig. 22. UV colours of Models A (top panel) and B (bottom panel) as a function of the red-shift. The cosmological parameters are $H_{0}=80 \mathrm{~km} \mathrm{~s}^{-1} \mathrm{Mpc}^{-1}, q_{0}=0$ For purpose of comparison the classical colour $(V-I), C_{55 \mathrm{~W}-81 \mathrm{~W}}$, is also shown. The two models differ in the kind of star formation. See the text for more details

Gyr) and very low efficiency of star formation $(\nu=0.5)$. This model never undergoes the galactic wind phase and thus has ever continuing star formation. It corresponds to a sort of spiral galaxy.

In order to assess the ability of UV colours in determining the age of galaxies we examine the variation as a func-

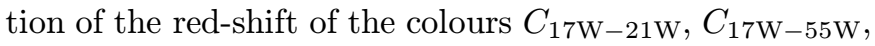

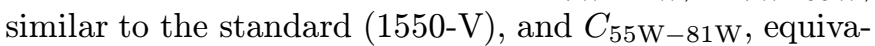
lent to the Johnson-Cousins $(V-I)$.

Preliminary to any other consideration is to check whether the age dependence of the integrated colours $C_{17 \mathrm{~W}-21 \mathrm{~W}}$ and $(1550-\mathrm{V})$ is somewhat affected by the 


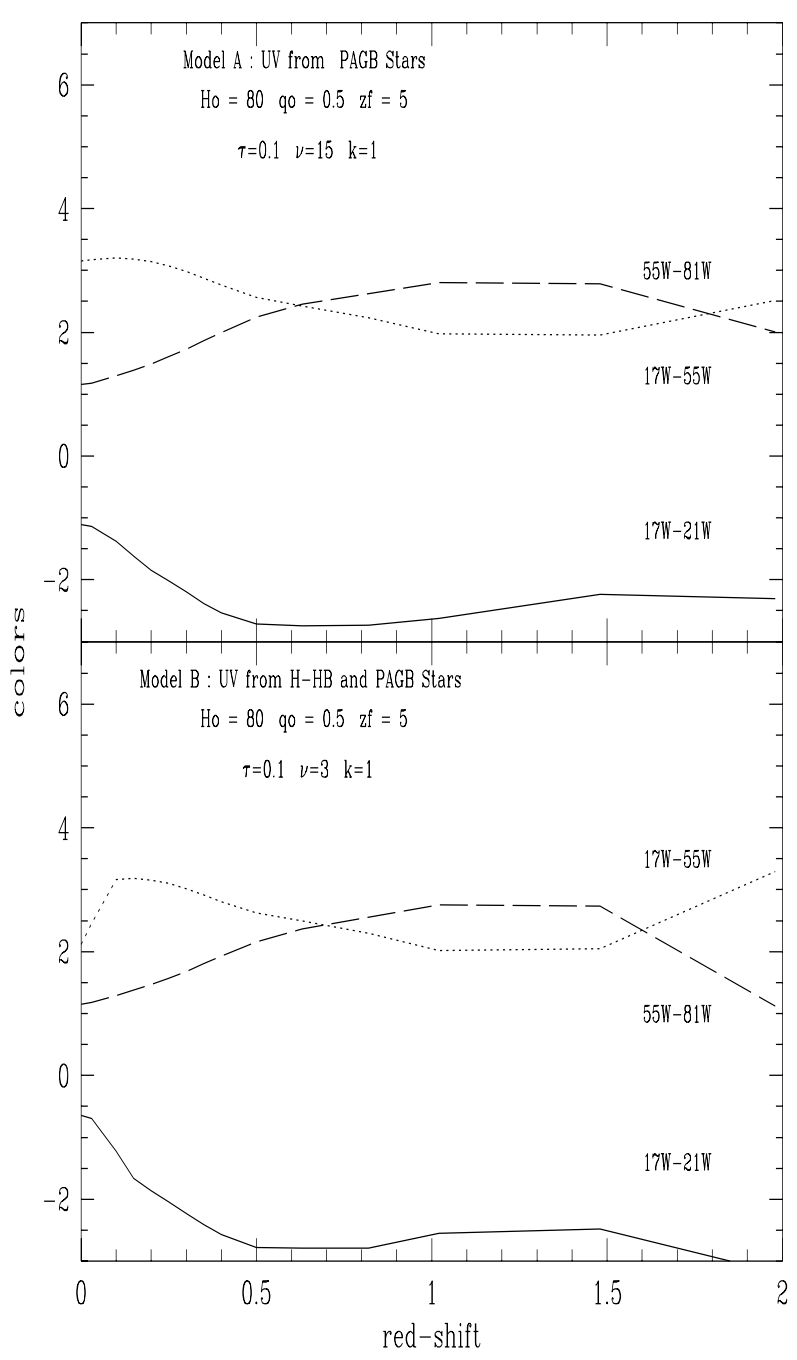

Fig. 23. UV colours of Models A (top panel) and B (bottom panel) as a function of the red-shift. The cosmological parameters are $H_{0}=80 \mathrm{~km} \mathrm{~s}^{-1} \mathrm{Mpc}^{-1}, q_{0}=0.5$ For purpose of comparison the classical colour $(V-I), C_{55 \mathrm{~W}-81 \mathrm{~W}}$, is also shown. The two models differ in the kind of star formation. See the text for more details

particular choice for the cosmological parameters $H_{0}$ and $q_{0}$. This is shown in the two panels of Fig. 18, where only ages older than 3 Gyr are considered. As expected, but for the maximum age of the galaxies indicated by the vertical arrows, the colour relations are the same at varying $H_{0}$ and $q_{0}$.

Examining the color-age relations in more detail, we notice:

- In Model A, the colour (1550-V) run almost flat up to age of about 11.5 Gyr at which P-AGB stars become important sources of UV radiation and then gently gets bluer, whereas the color $C_{17 \mathrm{~W}-21 \mathrm{~W}}$ has a minimum at the age of 11.5 Gyr.

- In Model B both colours get suddenly bluer at the age of about 5.6 Gyr and remain nearly constant afterward. The sudden variation simply reflects the appearance of H-HB and AGB-manqué stars, whereas the nearly constant colours afterward reflect the fact that the emission by $\mathrm{H}-\mathrm{HB}$ and AGB-manqué stars masks that by P-AGB and normal HB stars.

- In Model C owing to the ever continuing star formation, the colours are always very blue and nearly constant.

How these signatures in the rest-frame evolution of the colours for the three models will be affected by the cosmological distorsion of the ISED ?

The topic is addressed showing in the series of Figs. 19, 20, 22, and 23 the colour red-shift evolution for different choices of $H_{0}$ and $q_{0}$.

We begin by assuming $H_{0}=50$ and $q_{0}=0$ and in Fig. 19 we compare Model A (top panel) with Model B (central panel), i.e. the effect of different sources of UV radiation (P-AGB stars alone versus $\mathrm{P}-\mathrm{AGB}$ plus $\mathrm{H}-\mathrm{HB}$ and AGB-manqué stars), and Models $\mathrm{B}$ (central panel) with model $\mathrm{C}$ (bottom panel), i.e. the extreme avenues of star formation: an early burst and ever continuing activity.

Looking at the three panels of Fig. 19, we notice that the colours $C_{17 \mathrm{~W}-21 \mathrm{~W}}$ and $C_{17 \mathrm{~W}-55 \mathrm{~W}}$ of Model B are somehow anti-correlated. While $C_{17 \mathrm{~W}-21 \mathrm{w}}$ gets redder at increasing red-shift, $C_{17 \mathrm{~W}-55 \mathrm{~W}}$ gets bluer. At $z \simeq 1$ corresponding to the age of $5.6 \mathrm{Gyr}$ at which the $\mathrm{H}-\mathrm{HB}$ and AGB manqué stars start shining in the UV, both colors suffer from a sharp change reversing their trend, and run smooth afterward.

In contrast the UV colours of Model A (only PAGB stars present) have a different behaviour: the colour $C_{17 \mathrm{~W}-55 \mathrm{~W}}$, equivalent to $(1550-\mathrm{V})$, runs smooth as expected from the gradual appearance of the P-AGB stars, whereas the colour $C_{17 \mathrm{~W}-21 \mathrm{~W}}$ shows a marked dip at $z \simeq 0.4$, which does not find immediate correspondence in any particular stage of the rest-frame evolution $(z \simeq 0.4$ means an age of $10.8 \mathrm{Gyr}$ ). Since this value is close to age at which the rest-frame colour $C_{17 \mathrm{~W}-21 \mathrm{w}}$ has the bluest value one could argue that this reversal of the trend causes the dip in the colour-red-shift relation. The discussion below will clarify that this is not the explanation.

Even more noticeable is the different behaviour of the colours of Model $\mathrm{C}$ with continuing star formation. At $z=0$ all the UV colours are extremely blue as expected due to the combined effect of star formation and occurrence of $\mathrm{P}-\mathrm{AGB}, \mathrm{H}-\mathrm{HB}$ and AGB-manque stars (the latter two are present because of the high mean and maximum metallicity of the model). Going back in time, the colour $C_{17 \mathrm{~W}-55 \mathrm{~W}}$ gets first bluer and then redder (the bluest value is at $z=\simeq 0.6$, whereas the colour $C_{17 \mathrm{~W}-21 \mathrm{~W}}$ has its reddest value at $z \simeq 1.2$. This trends cannot be straightforwardly related to any particular feature in the 
rest-frame evolution of the colours because ongoing star formation wipes out the signature of the old components (cf. Fig. 18).

Passing from $q_{0}=0$ to $q_{0}=0.5$ and keeping $H_{0}=50 \mathrm{~km} \mathrm{~s}^{-1} \mathrm{Mpc}^{-1}$ (Fig. 20), the discontinuity in the

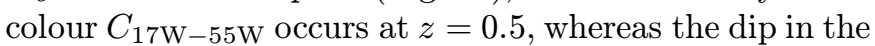

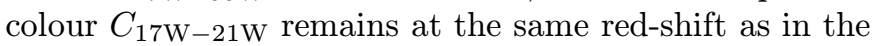
previous case. Since for $q_{0}=0.5$, a red-shift $z \simeq 0.4$ translates into an age of about $7 \mathrm{Gyr}$, at which no signature is found in the rest-frame colour evolution is found (cf. Fig. 18), the constancy of the red-shift at which the dip is found strongly argues against any possible evolutionary interpretation of this latter.

In order to prove this statement, we examine how the ISED of Models A and B vary with age. This is shown in Fig. 21, which displays for three selected values of the age the rest-frame ISED of Model A and $\mathrm{B}$ in the wavelength region $0<\lambda<4000 \AA$. In Model A (only P-AGB dominating the UV flux), the shape of the ISED does not vary with time at any significant level over a large range of ages. The only effect to be noticed is that the level of the flux in the $1000<\lambda<2000 \AA$ region increases with the age. In contrast in Model B both the shape and the flux level significantly vary with the age passing from 5 Gyr to older galaxies. The reason of it is easy to understand. In Model A, over the age range under consideration the source of UV flux are P-AGB of nearly identical $T_{\text {eff }}$ with nearly identical ISED. In contrast, in Model B both P-AGB and H-HB stars intervene, whose $T_{\text {eff }}$ and resulting ISED greatly vary with time. Therefore, in the case of Model A the minimum in the colour $C_{17 \mathrm{~W}-21 \mathrm{~W}}$ simply reflects the gradual effect of the red-shift transferring radiation from one pass-band to the other. Since there is no age effect, the red-shift at which the reversal of the colour trend occurs does not depend on the $H_{0}$ and $q_{0}$. In the case of Model B the onset of the H-HB and AGB-manqué stars changes both the shape and intensity of the ISED. Therefore a real age term is present whose effect is to make the colour-red-shift relation vary with $H_{0}$ and $q_{0}$.

As a result of this analysis, only the colour $C_{17 W-55 W}$ might be a promising age indicator.

Changing the Hubble constant $H_{0}$ from 50 to $80 \mathrm{~km} \mathrm{~s}^{-1} \mathrm{Mpc}^{-1}$ (Figs. 22 and 23) we get similar results for $q_{0}=0$, but very different ones for $q_{0}=0.5$. In this latter case, the maximum galaxy ages are so young that the contribution from the above stellar sources to the UV radiation are almost lost. Although the red-shift de-

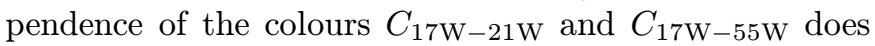
no longer allow us to discriminate between $\mathrm{H}-\mathrm{HB}$ and $\mathrm{P}$ AGB dominated UV emission, its remarkable difference with respect to those for the other values of $H_{0}$ and $q_{0}$ might turn out to be useful to set constraints on the cosmological parameters rather than on the age.

Although promising, the results of the above analysis could be somewhat weakened by two remarks. First, the HST UV filters suffer from a strong/visible red leak.
It is very plausible that they might be sensitive to other kind of emissions such as that caused by residual bursts of star formation still emitting in the visible, nebular emissions... which could make the age dependence of the colour $C_{17 \mathrm{~W}-55 \mathrm{~W}}$ less significant. Second, the intensity of the UV emission is known to vary with the distance from the galactic centre, being more intense in the nuclear than in the external regions. To this aim see for instance the change of UV colour $(1500-2200)$ in NGC 1399, M 31, and M 81 with the radial distance (O'Connell 1992). Disentangling whether this is an age or a metallicity effect is a cumbersome affair. Certainly it reflects different types of stars generating the UV flux. Bressan et al. (1996) studying the narrow band indices $H_{\beta}$ and $<\mathrm{MgFe}>$ of the Gonzales (1993) sample of galaxies (ellipticals) suggest that the vast majory of these have the nuclear region younger and more metal-rich than the peripheral regions. Unless the central regions of these galaxies have sufferend from a very recent episode of star formation, they are the natural site to look at in which H-HB and AGBmanqué stars can be found, whose UV emission should vary with time as shown in Fig. 18. In the remaining part of the galaxy the UV emission should be generated by the ever present P-AGB stars. Going to higher and higher redshifts, with a given aperture of the detecting instument, a larger volume of the galaxy is sampled so that the different sources of UV radiation are more and more mixed together, thus diluting the signatures of each component.

Despite the above remarks, what we learn from these examples is that using observations in the UV at different red-shifts, we can perhaps pin down the dominant source of UV radiation, constrain the relation between $H_{0}, q_{0}$ and $z_{\text {for }}$ and in principle determine the age of galaxies. However, it is beyond the aims of this paper to further investigate the problem, which is left to future studies.

\section{Conclusions}

We have calculated extensive grids of magnitudes and colours for stars of any spectral type in several pass-bands of the HST WFPC2 and FOC, as well as in the JohnsonCousins system. The analysis has been made using the library of stellar spectra calculated by Kurucz (1992) and filtering the spectral energy distribution with the convolution of the pass-band and quantum efficiency of the WFPC2 and/or FOC and the telescope assembly. The HST magnitudes are presented by means of tabulations of Bolometric Corrections $\left(B C_{\Delta \Lambda}^{\mathrm{HST}}\right)$ as a function of the metallicity, $T_{\text {eff }}$, and gravity which allow us to convert the bolometric luminosity of a star into the HST magnitudes and colours.

We have also presented integrated magnitudes and colours as a function of the age for SSPs obeying the Salpeter initial mass function.

Furthermore, we have analyzed the CMD of a typical sample of stars containing both old and young objects. 
The results are presented both for the Johnson-Cousins and the HST-WFPC2 photometric systems pointing out the distortion engendered in this latter by the non monotonic colour- $T_{\text {eff }}$ relation for the extreme UV pass-bands. These simulations are intended to call the reader attention on the peculiarity inherent to the UV pass-bands and on the unexpected appearance of the otherwise familiar CMDs in those HST UV pass-bands.

Finally, we have presented the colour evolution of elliptical galaxies as a function of the red-shift in order to check whether the onset of the P-AGB and/or H-HB and AGB-manqué stars as old sources of UV radiation can be detected in suitable UV colours at certain values of the red-shift. We find that the colour -red-shift relations exhibit signatures that can be traced back to particular evolutionary stages of the underlying stellar populations, whose nature and age are known at certain degree of confidence. Furthermore the detailed colour-red-shift relation is found to depend also on the cosmological parameters. If these predictions are confirmed by observational data they will allow us to infer the type of source of UV radiation, and constrain the galaxy age and cosmological parameters $H_{0}$ and $q_{0}$.

Detailed tabulations of isochrones and $B C_{\Delta \lambda}^{\mathrm{HST}}$ for other chemical compositions and/or pass-bands are available from the authors upon request.

Acknowledgements. This work was supported by Italian Space Agency (ASI) and the Ministry of University, Scientific Research, and Technology of Italy (MURST).

\section{References}

Baade W., 1951, Publ. Univ. Mich. Obs. 10, 7

Bell R.A., VandenBerg D.A., 1987, ApJS 63, 335

Bertelli G., Bressan A., Chiosi C., Fagotto F., Nasi E., 1994, A\&AS 106, 275

Bertola F., Burstein D., Buson L., Chiosi C., Di Serego Alighieri S., 1995, ApJ 438, 680

Bica E., Alloin D., Schmidt A.A., 1990, A\&A 228, 23

Bower R.G., Lucey J.R., Ellis R.S., 1992, MNRAS 254, 601

Bressan A., 1996 (preprint)

Bressan A., Chiosi C., Fagotto F., 1994, ApJS 94, 63

Bressan A., Chiosi C., Tantalo R., 1996, A\&A 311, 425

Brown T.M., Ferguson H.C., Davidsen A.F., 1995, ApJ 454, L15

Bruzual G., 1992, in The Stellar Populations of Galaxies, Barbuy B. and Renzini A. (eds.). Dordrecht: Kluwer Academic Publishers, p. 311

Bruzual G., Charlot S., 1993, ApJ 405, 538

Burstein D., Bertola F., Buson L.M., Faber S.M., Lauer T.R., 1988, ApJ 328440

Bushouse H., 1993, Synphot User Guide, 81

Carraro G., Girardi L., Bressan A., Chiosi C., 1996, A\&A (in press)

Charlot S., Bruzual G., 1991, ApJ 367, 126

Chiosi C., Bertelli G., Bressan A., 1988, A\&A 196, 84

Chiosi C., Bertelli G., Meylan G., Ortolani S., 1989, A\&A 219, 167
Dorman B., O'Connell R.W., Rood R.T., 1995, A\&A 442, 105

Dorman B., Rood R.T., O'Connell R.W., 1993, A\&A 419, 516

Edvardsson B., Bell R.A., 1989, MNRAS 238, 1121

Ferguson H.C., Davidsen A.F., 1993, ApJ 408, 92

Ferguson H.C., Davidsen A.F., Kriss G.A., et al., 1991, ApJ 382, L69

Fluks M.A., Plez B., Thé P.S., de Winter D., Westerlund B.E., Steenman H.C., 1994, A\&AS 105, 311

Fusi-Pecci F., Cacciari C., 1991, in New Windows to the Universe, Sanchez F. and Vasquez M. (eds.). Cambridge, Cambridge Univ. Press, p. 364

Gallagher J.S., Mould J.R., 1981, ApJ 244, L3

Girardi L., Chiosi C., Bertelli G., Bressan A., 1995, A\&A 298, 87

Gonzales J.J., 1993, Ph.D. Thesis, University of California, Santa Cruz

Greggio L., Renzini A., 1990, ApJ 364, 35

Harris H.C., Hunter D.A., Baum W.A., Jones J.H., Kreidl T.J., 1988, in Report to the Space Telescope Science Institute on Ground-Based Observations for the WF/PC Photometric Calibration, Lowell Observatory, Flagstaff

Hesser J.E., Harris W.E., VandenBerg D.A., Allwright J.W.B., Shott P., Stetson P.B., 1987, PASP 99, 739

Hodge P.W., 1973, AJ 182, 671

Holtzman J.A., Burrows C.J., Casertano S., et al., 1995a, PASP 107, 1065

Holtzman J.A., Hester J.J., Casertano S., et al. 1995b, PASP 107,156

King I., et al., 1992, ApJ 397, L35

Kurucz R.L., 1992, Rev. Mex. AA 23, 45

Lee Y-W., 1994, ApJ 423, 248

Marigo P., Bressan A., Chiosi C., 1996, A\&A 313, 545

McClure R.D., VandenBerg D.A., Bell R.A., Hesser J.E., Stetson P.B., 1987, AJ 93, 1144

Mould H., Kristian J., da Costa G., 1983, ApJ 270, 471

Mould H., Kristian J., da Costa G., 1984, ApJ 278, 575

Nota A., Jedrezejewski R., Greenfield O., Hack W., 1994, HST Faint Object Camera Instrument Handbook (PostCOSTAR), version 5.0

O'Connell R.W, 1992, in The Stellar Populations of Galaxies, Barbuy B. and Renzini A. (eds.). Kluwer Academic Publishers: Dordrecht, p. 233

Pagel B.E.J., Simonson E.A., Terlevich R.J., Edmunds, M.G. 1992, MNRAS, 255, 325

Paltoglou G., Bell R.A., 1991, MNRAS 253, 449

Paresce F., et al., 1991, Nat 352, 297

Peletier R. F., 1993, A\&A 271, 51

Reimers D., 1975, Mem. Soc. Roy. Sci. Liege, $6^{\text {e }}$ Ser., 8, 369

Renzini A., Buzzoni A., 1986, in Spectral Evolution of Galaxies, Chiosi C., Renzini A. (eds.). Reidel, Dordrecht, p. 195

Renzini A., Voli M., 1981, A\&A 94, 175

Rocca-Volmerange B., Guiderdoni E., 1988, A\&AS 75, 93

Salpeter E.E., 1955, ApJ 121, 161

VandenBerg D.A., Bell R.A., 1985, ApJS 58, 561

Tantalo R., Chiosi C., Bressan A., Fagotto F., 1996, A\&A 311, 361

Vassiliadis E., Wood P.R., 1993, ApJ 413, 641

Weidemann V., 1987, A\&A 188, 74

Yi S., Demarque P., Oemler A., 1995, PASP 107, 273 